\title{
A novel synthesis of chiral guanidinium receptors and their use in unfolding the energetics of enantiorecognition of chiral carboxylates
}

Vinod D. Jadhav and Franz P. Schmidtchen*

Department of Chemistry, Technical University of Munich, Lichtenbergstrasse 4, 85747, Garching, Germany

schmidtchen@ch.tum.de

\section{Supporting information available}

1. General Methods and Materials

2. ${ }^{1} \mathrm{H}$ and ${ }^{13} \mathrm{C}$ NMR spectra of the compounds 2-7, 10-14, 17-18

3. Mass spectra (ESI-MS) of the compounds 2-7, 10-11 and HRMS of 2 and 11

4. Supporting material for entries depicted in table 1

5. Supporting material for entries depicted in table 2

6. Supporting material for entries depicted in table 3
$\mathrm{S} 2$

S3-S28

S29-S39

S40-S42

S43

S44-S45 


\section{The general methods and materials:}

All the experiments in organic solvents were performed under nitrogen atmosphere and monitored by HPLC. The HPLC-analysis were performed on Merck-Hitachi instruments L 6200A or 655 A-11 pump connected to Knauer L 4250 UV detector and Sedex light scattering detector and Kipps\&Zonen two channel recorder. The columns that were used in HPLC analysis are Phenomenex, Aqua $\mathrm{C}_{18}, 250 \times 4.60$ mm, $5 \mu$ column, Nucleodure-100-5 $\mathrm{C}_{8}$ ec column and Nucleodure-100-5 CN-RP column.

The solvents were purchased in pure analytical grade and distilled before use except for DMF which was purchased in anhydrous quality from Aldrich and acetonitrile which was purchased in HPLC-quality from Baker. THF was dried and purified by distillation under nitrogen from benzophenone and sodium. Aqueous solutions were prepared from deionized, glass distilled water. The solvents $\left(\mathrm{CH}_{2} \mathrm{Cl}_{2}, \mathrm{CH}_{3} \mathrm{CN}\right)$ were passed for drying through a small column of activated alumina directly into the reaction vessel. All other chemicals were purchased in reagent quality from commercial sources and used as received.

The column chromatography was performed on either silica gel $100(0.063-0.200 \mathrm{~mm})$ or activated alumina (neutral, activity grade-I).

Flash-Chromatography (MPLC) was performed using a $\mathrm{C}_{8}$ or $\mathrm{CN}$-modified silica gel in Michel-Miller columns connected to the Knauer HPLC-pump 364, and Knauer UV detector.

Purification of compounds by Solid Phase Extraction (SPE) was performed using commercially available SPE columns from Alltech (high capacity $\mathrm{C}_{18}$ ).

${ }^{1} \mathrm{H}$ and ${ }^{13} \mathrm{C}-\mathrm{NMR}$ spectra were recorded on a Brucker AM 250 or AC $360(\mathrm{MHz})$ instruments and were referenced with respect to the residual solvent peak for the deuterated solvent.

All the melting points were measured in open capillary tubes using a Fisher-Jones apparatus.

Mass spectra were obtained on Finnigan LQC: Electrospray-Ionization (ESI, HPLC-MS).

Elemental analyses were made by the microanalytical laboratory of the TU Munich.

Calorimetric titrations were performed on the Isothermal Titration Calorimeter MCS-ITC from Microcal Inc., Northampton, Massachusetts, USA. 


\section{2. ${ }^{1} \mathrm{H}$ and ${ }^{13} \mathrm{C}$ NMR spectra of the compounds $2-7,10-14,17-18$}

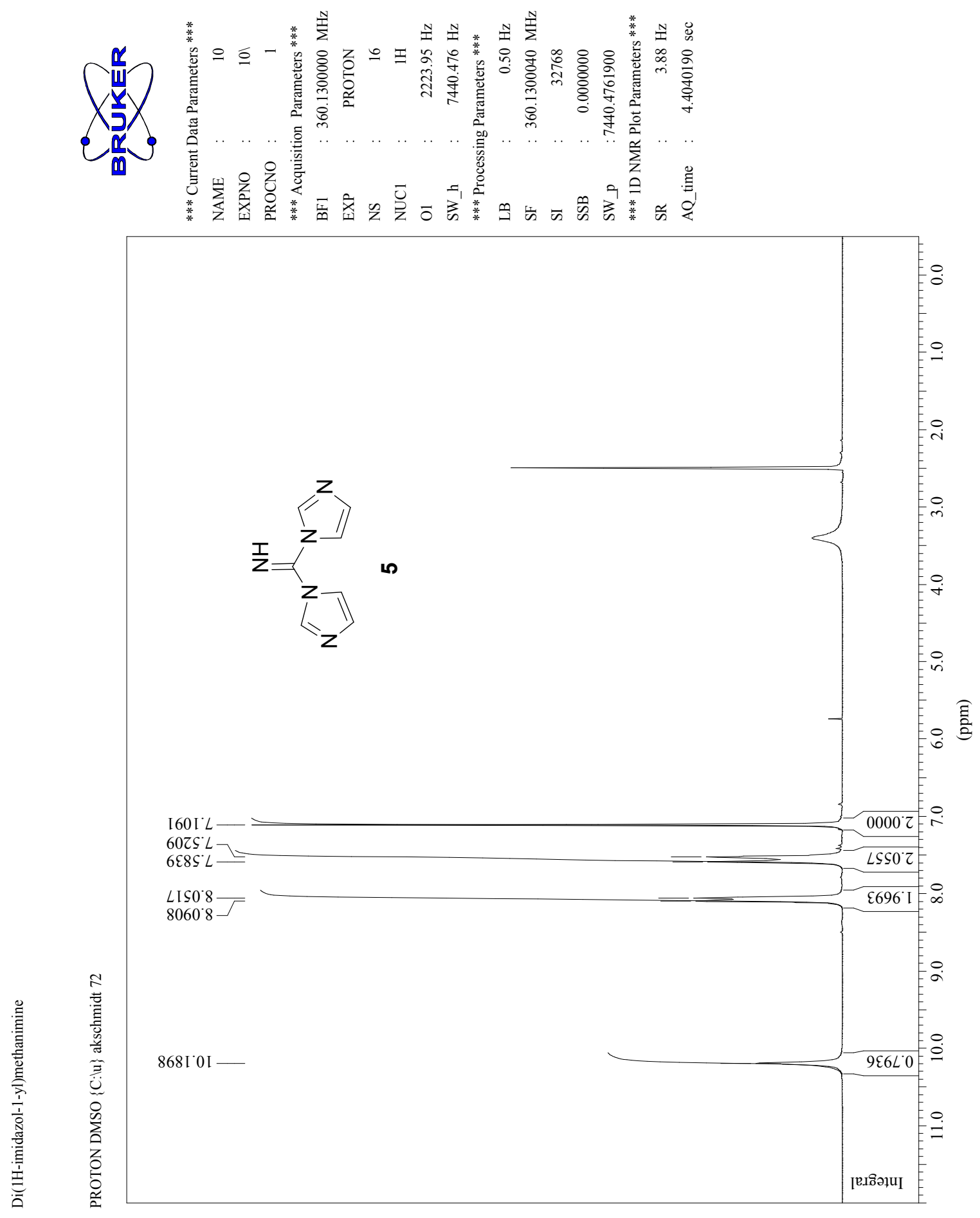



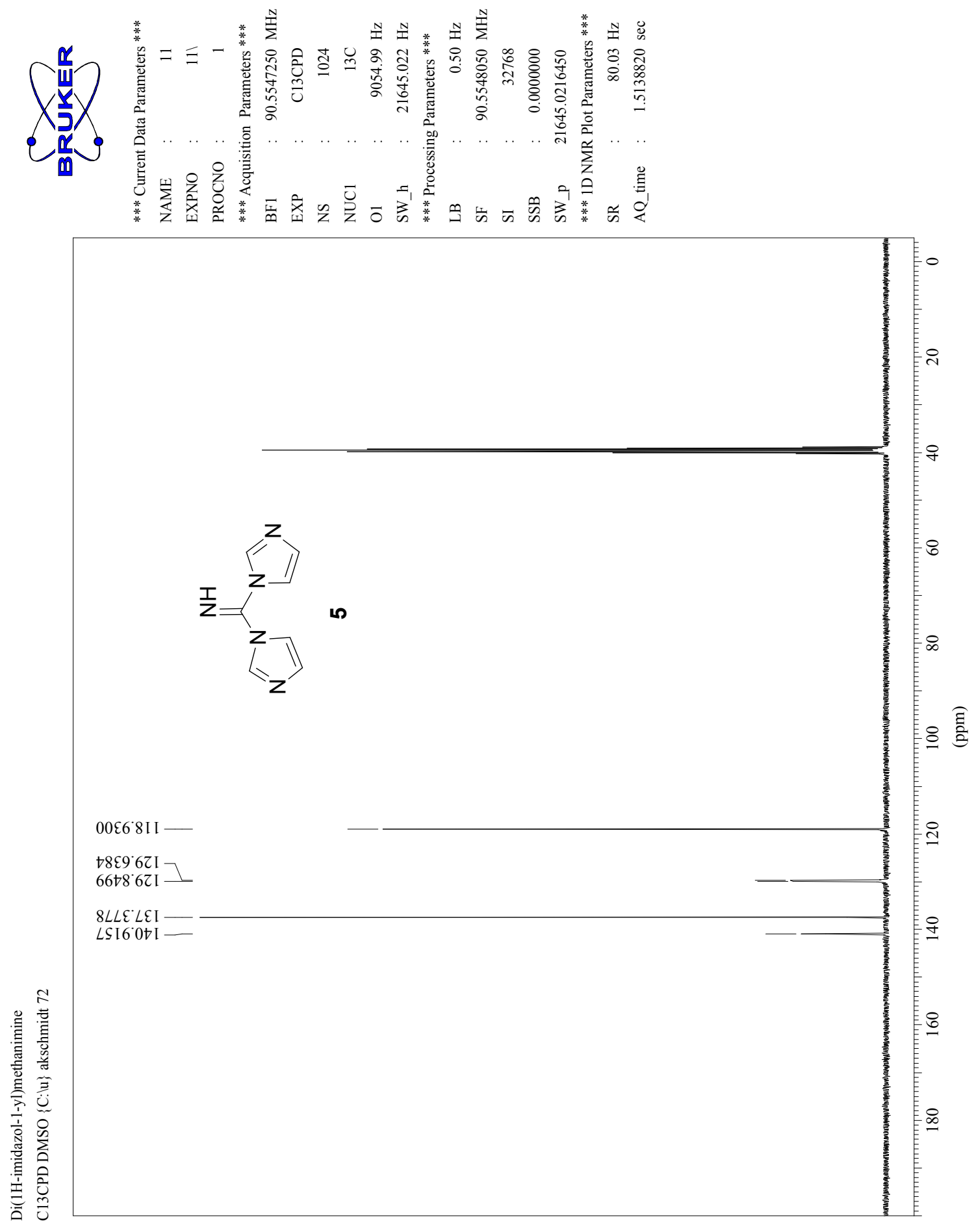

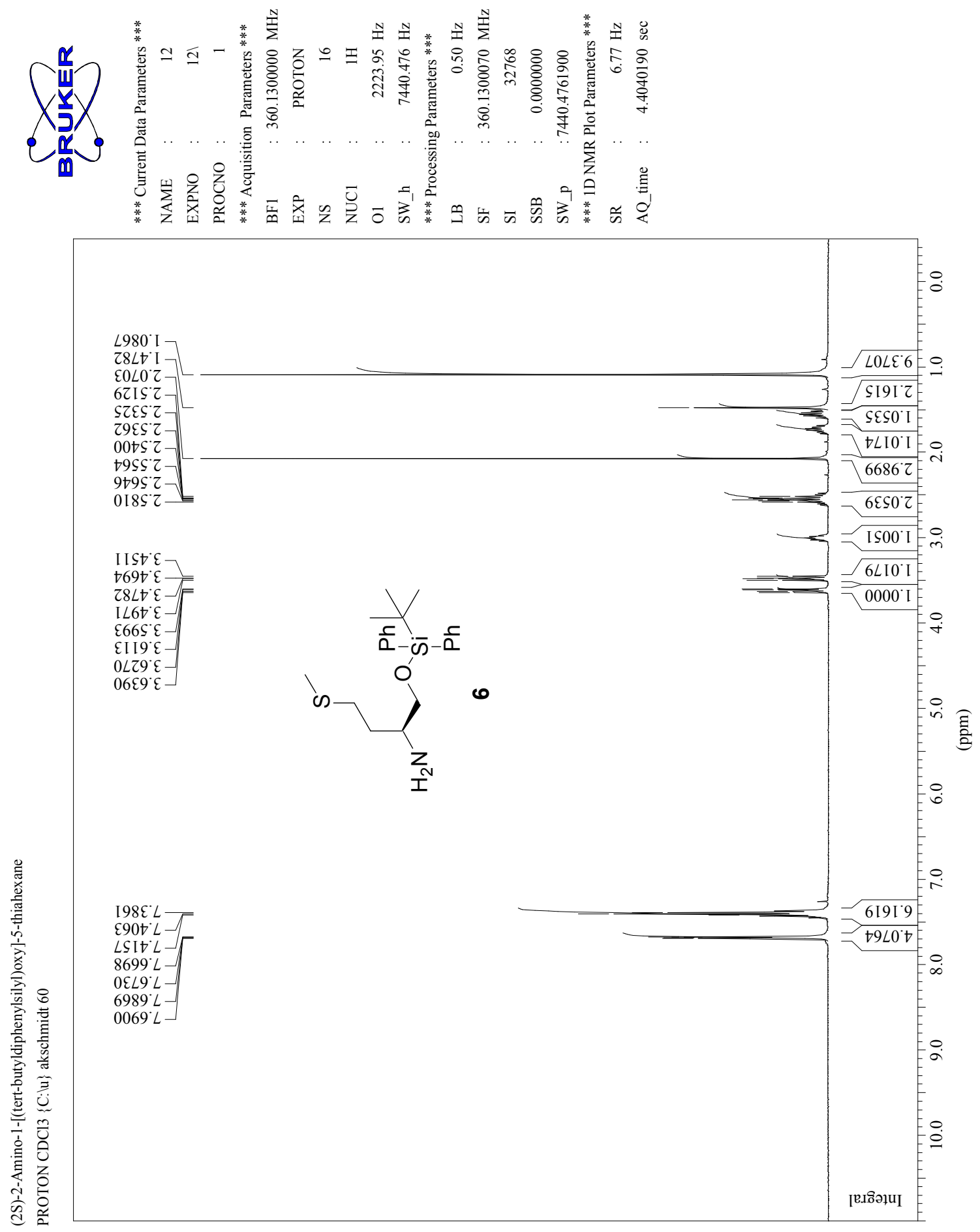

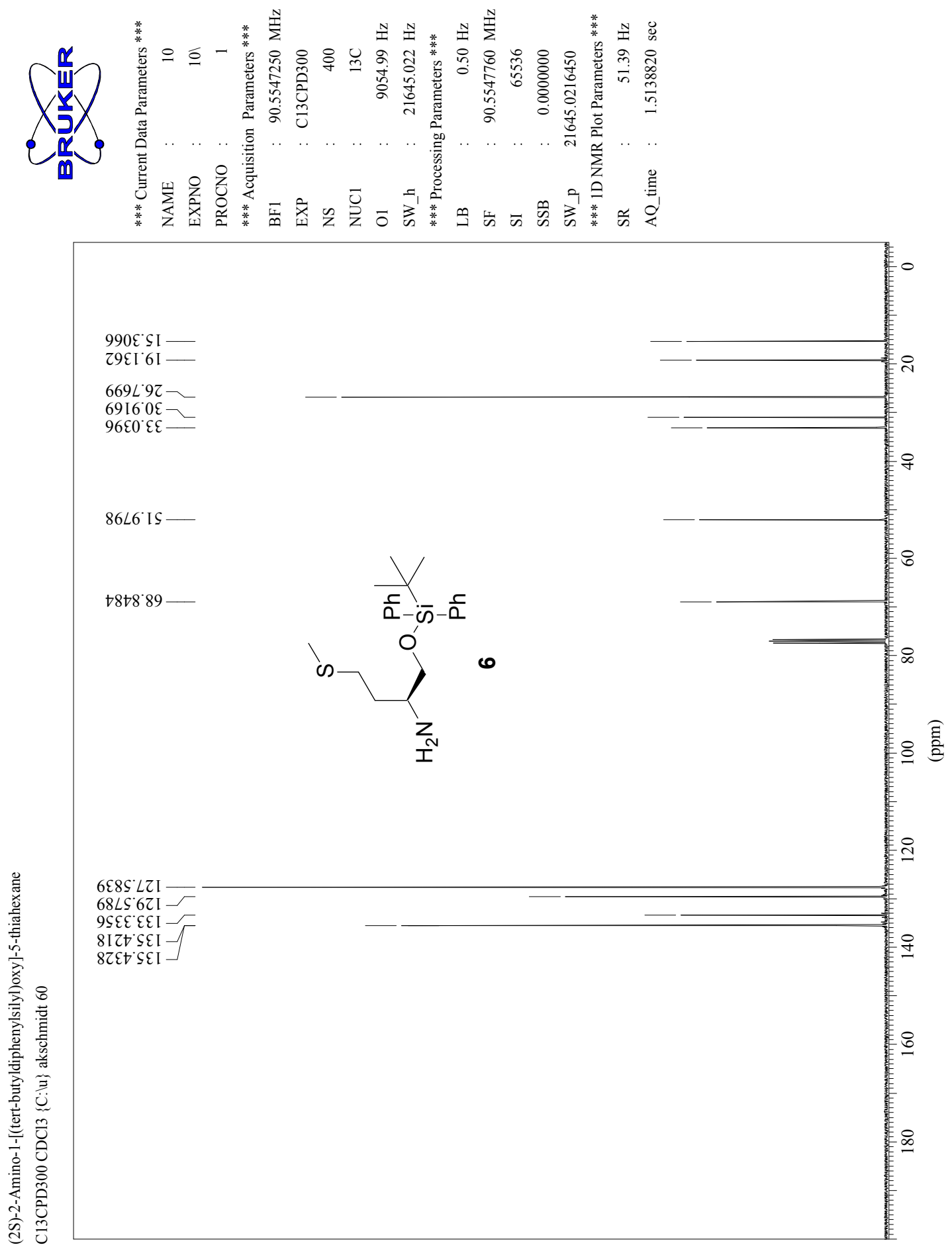

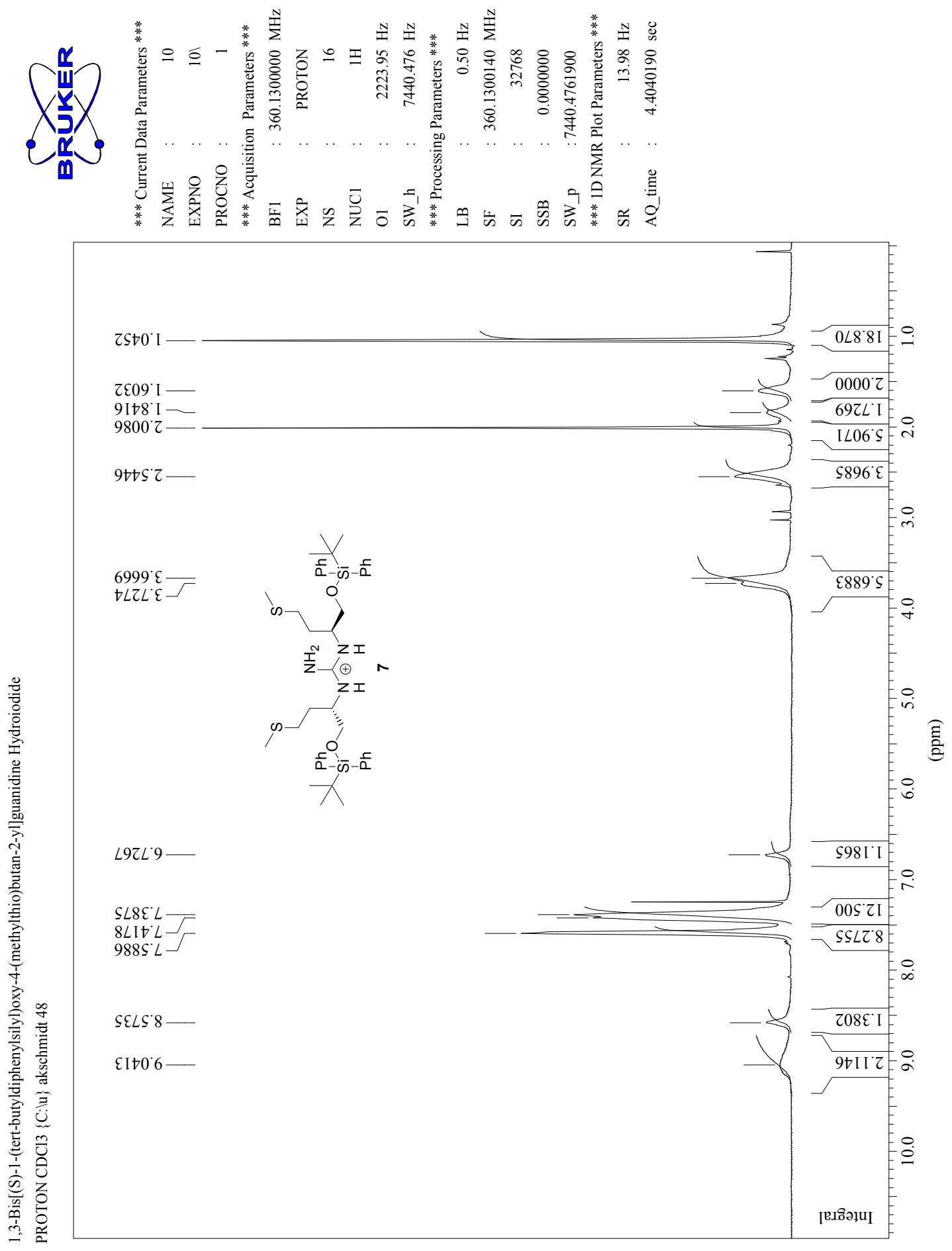


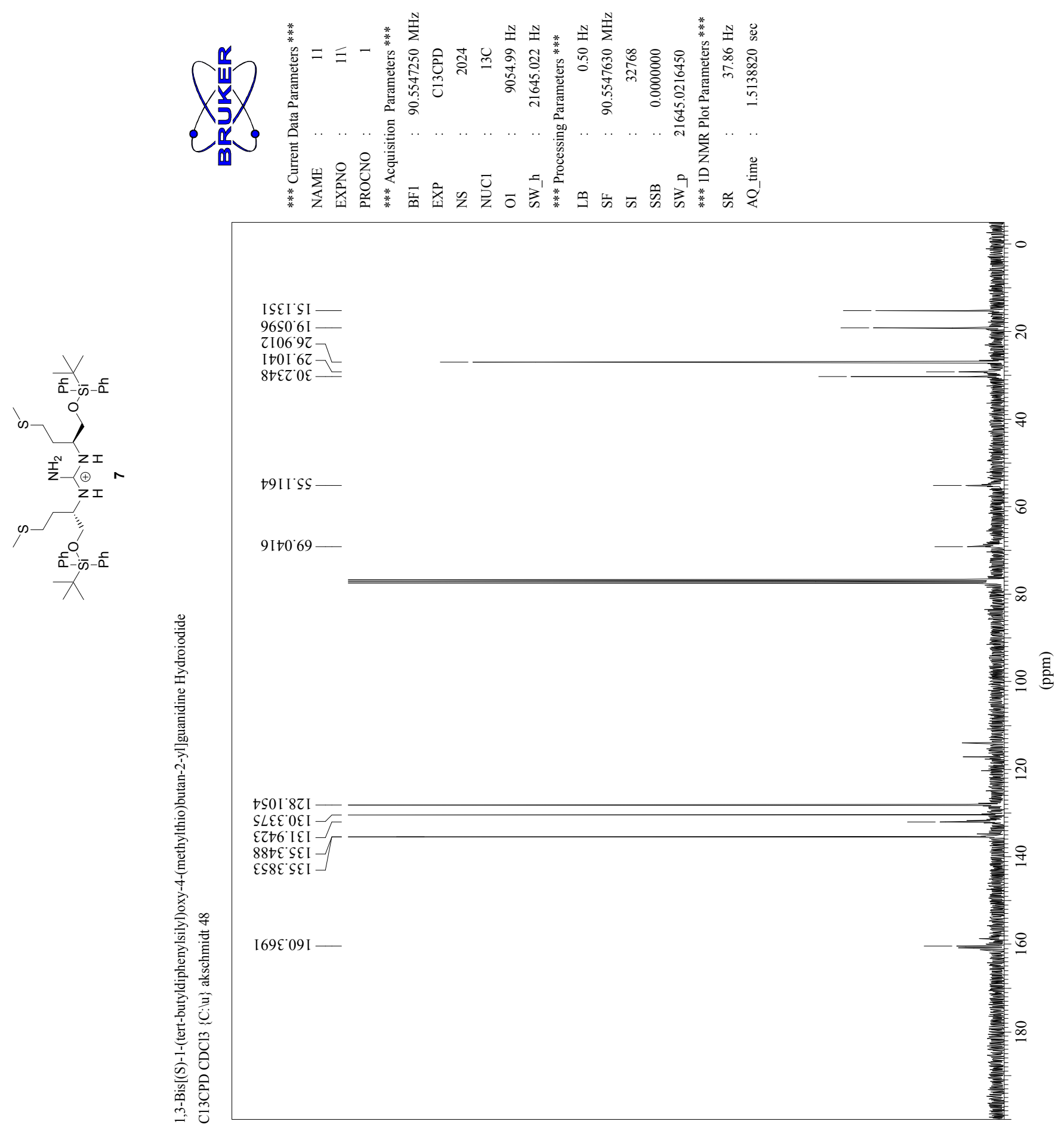



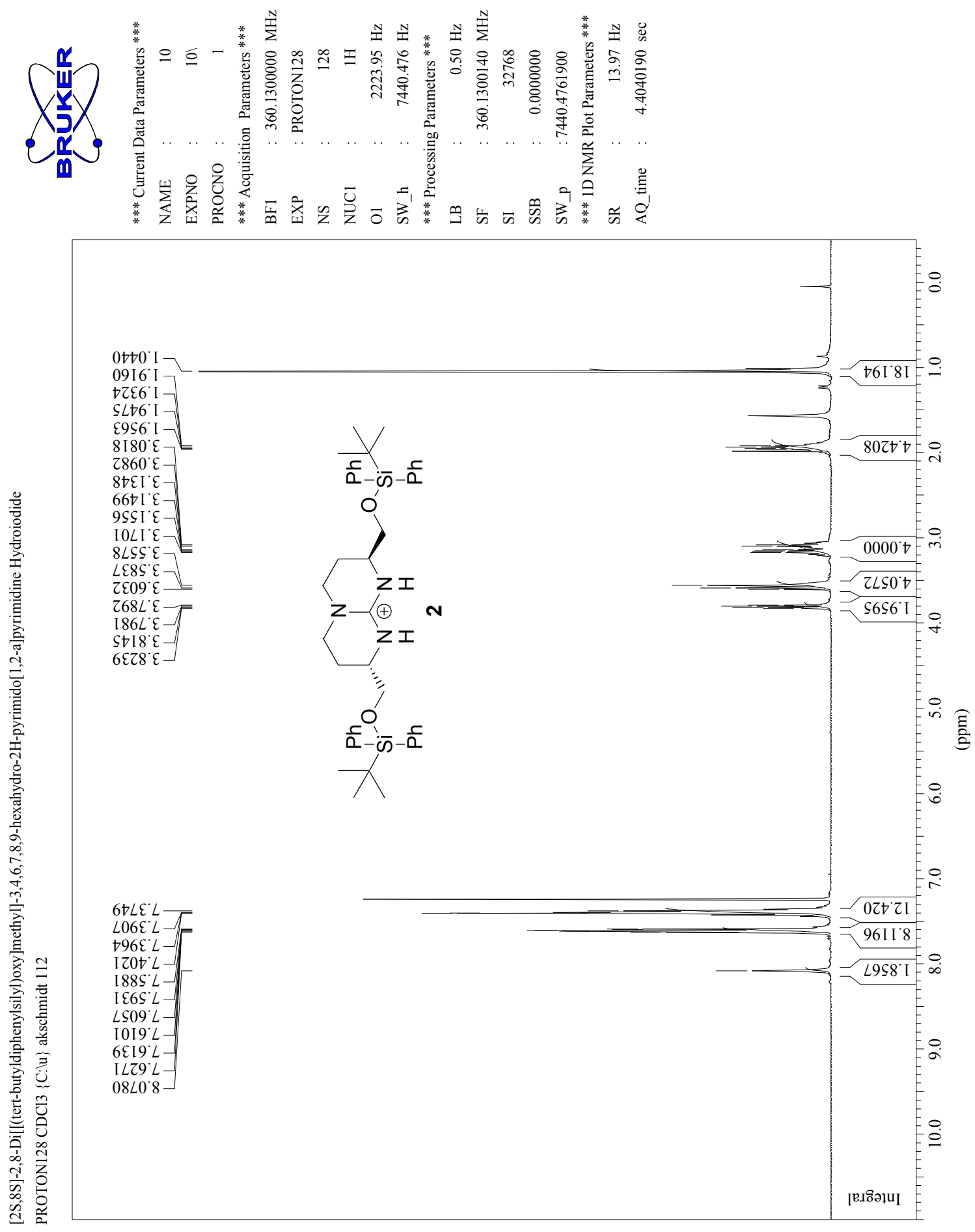

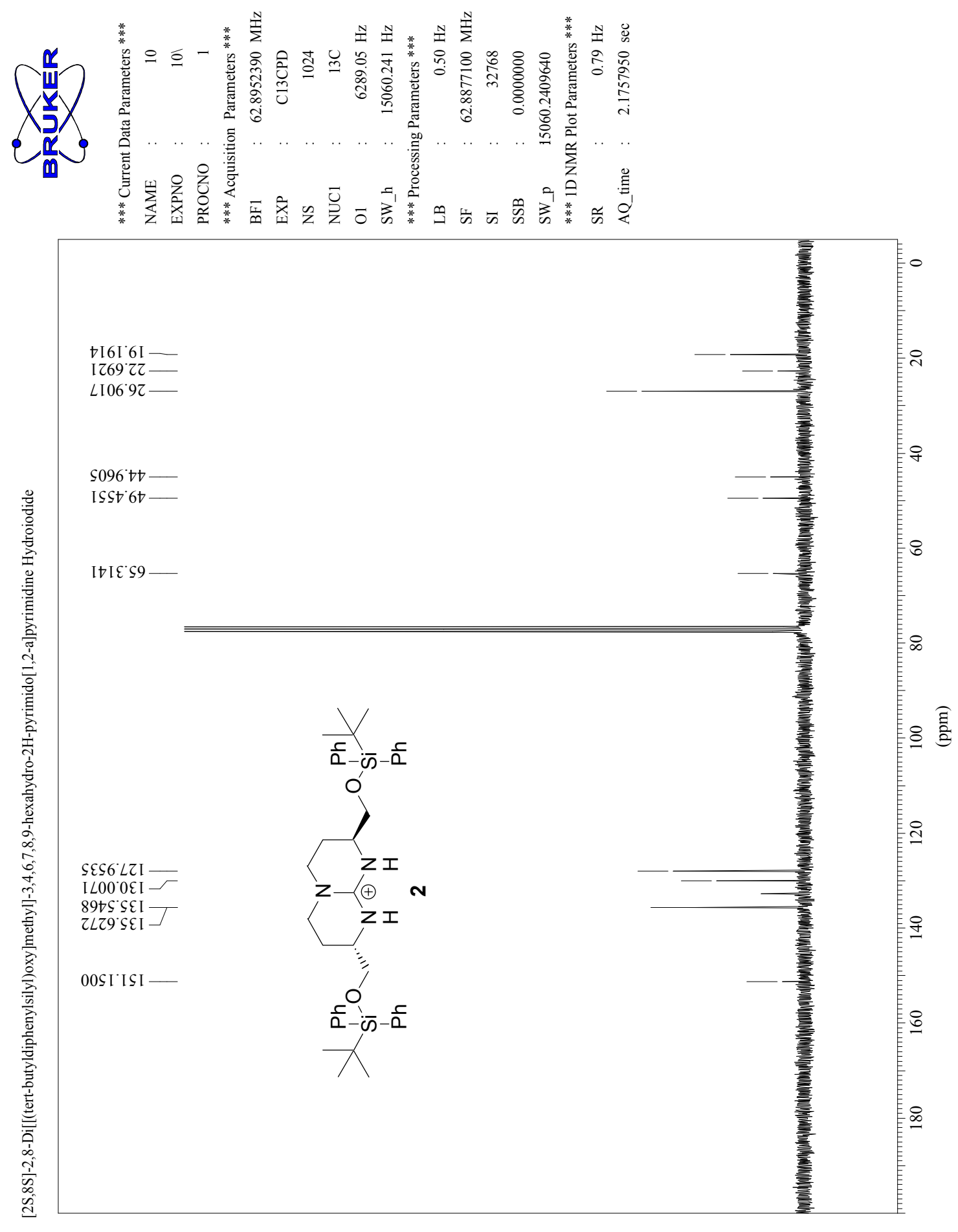

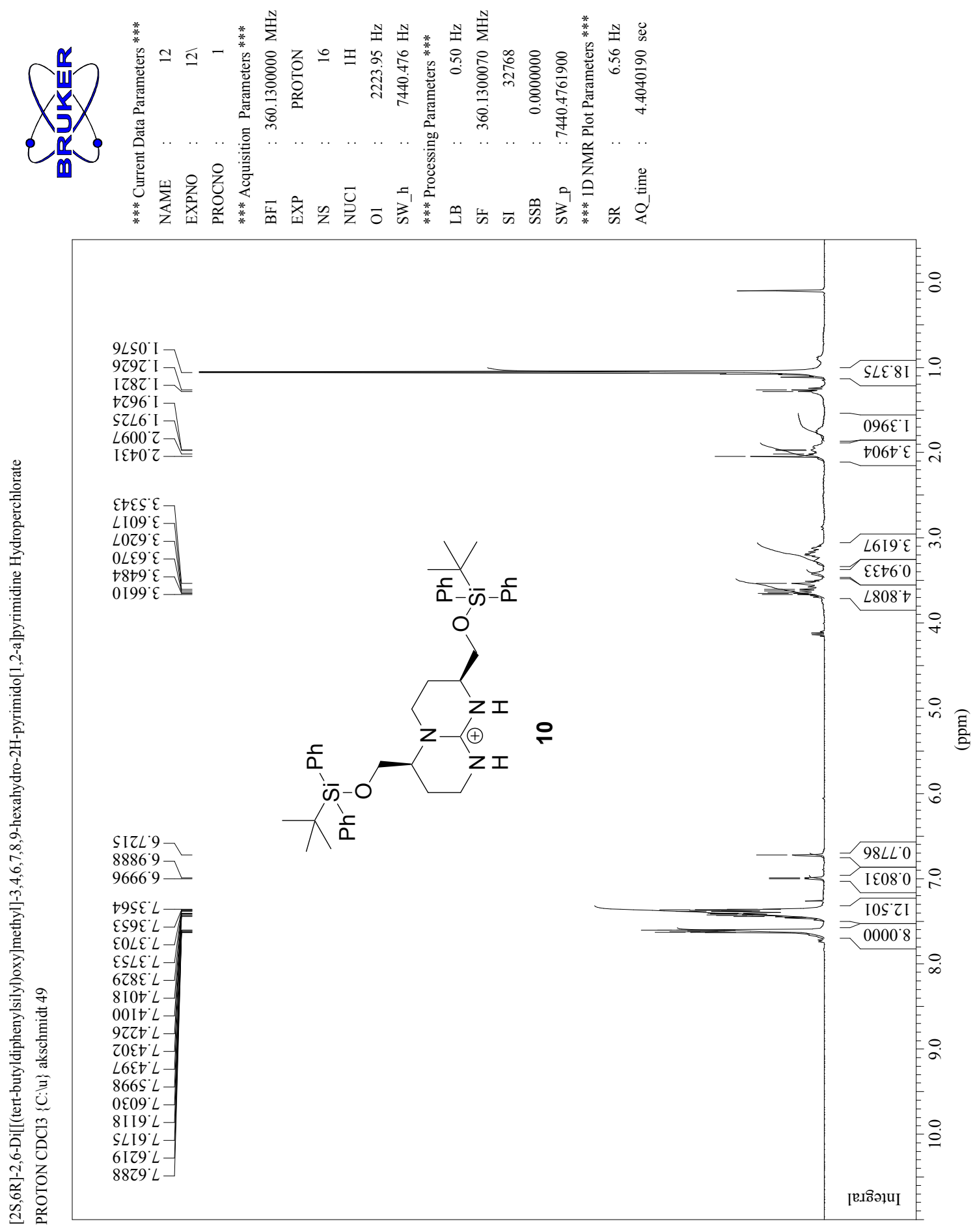

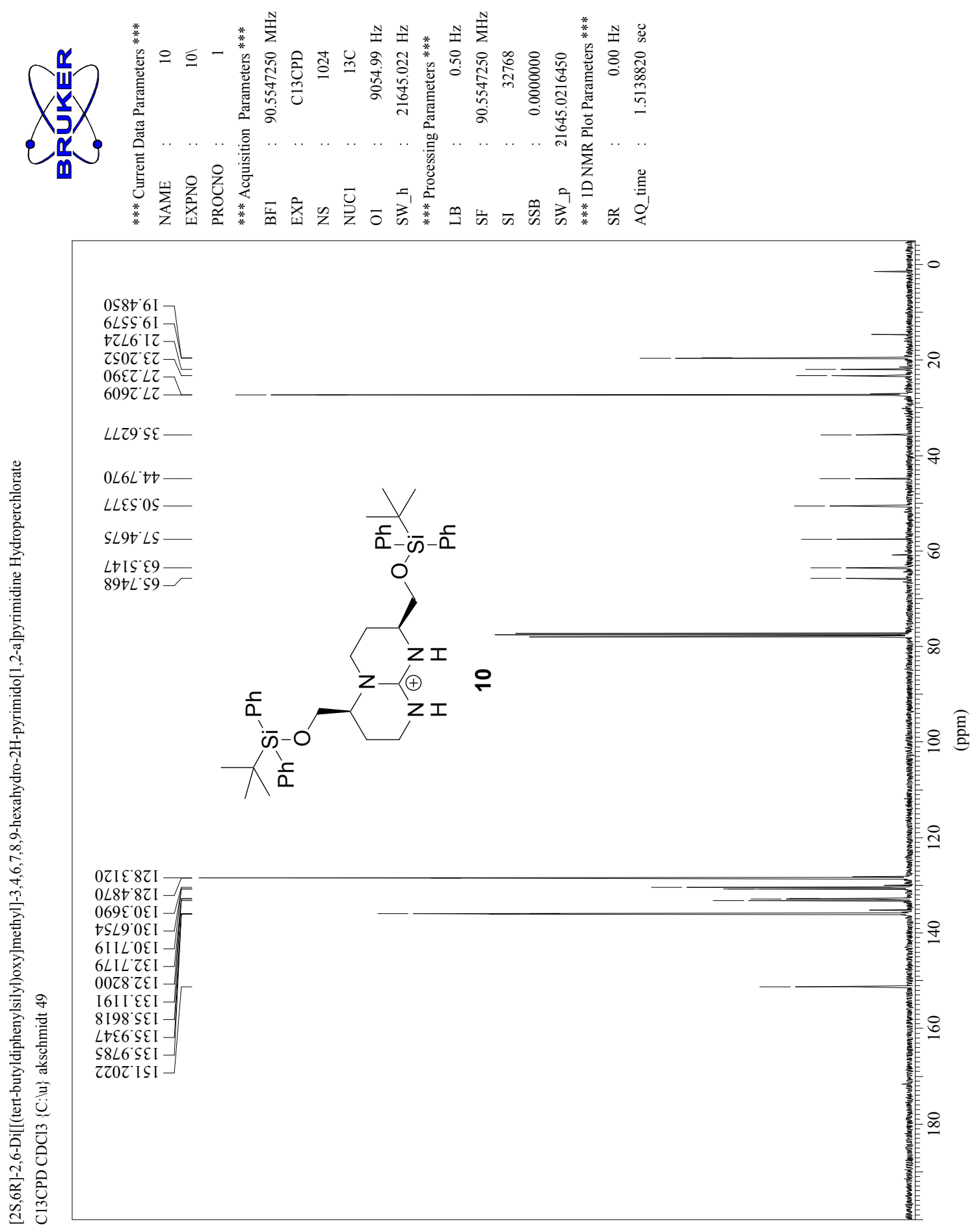

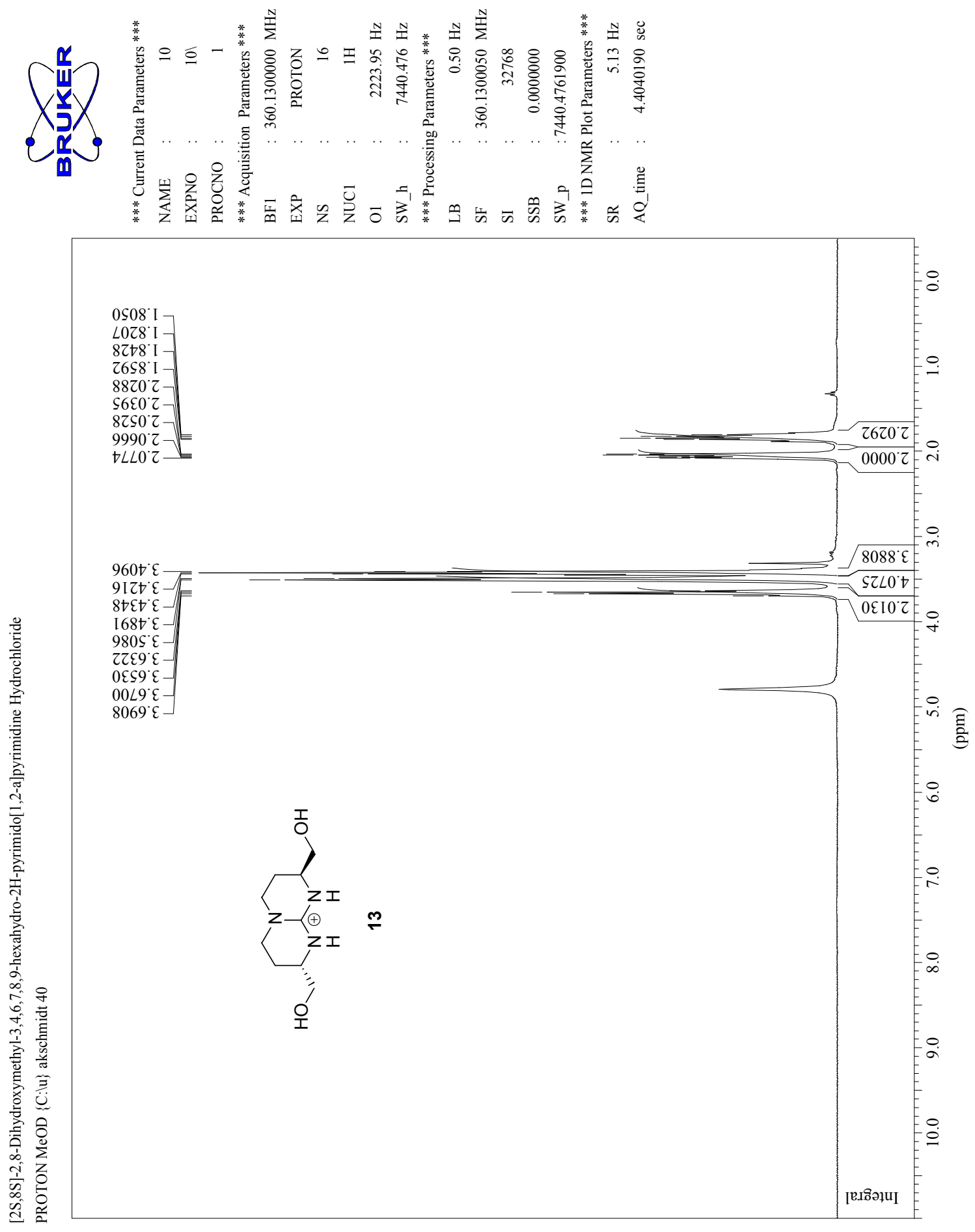

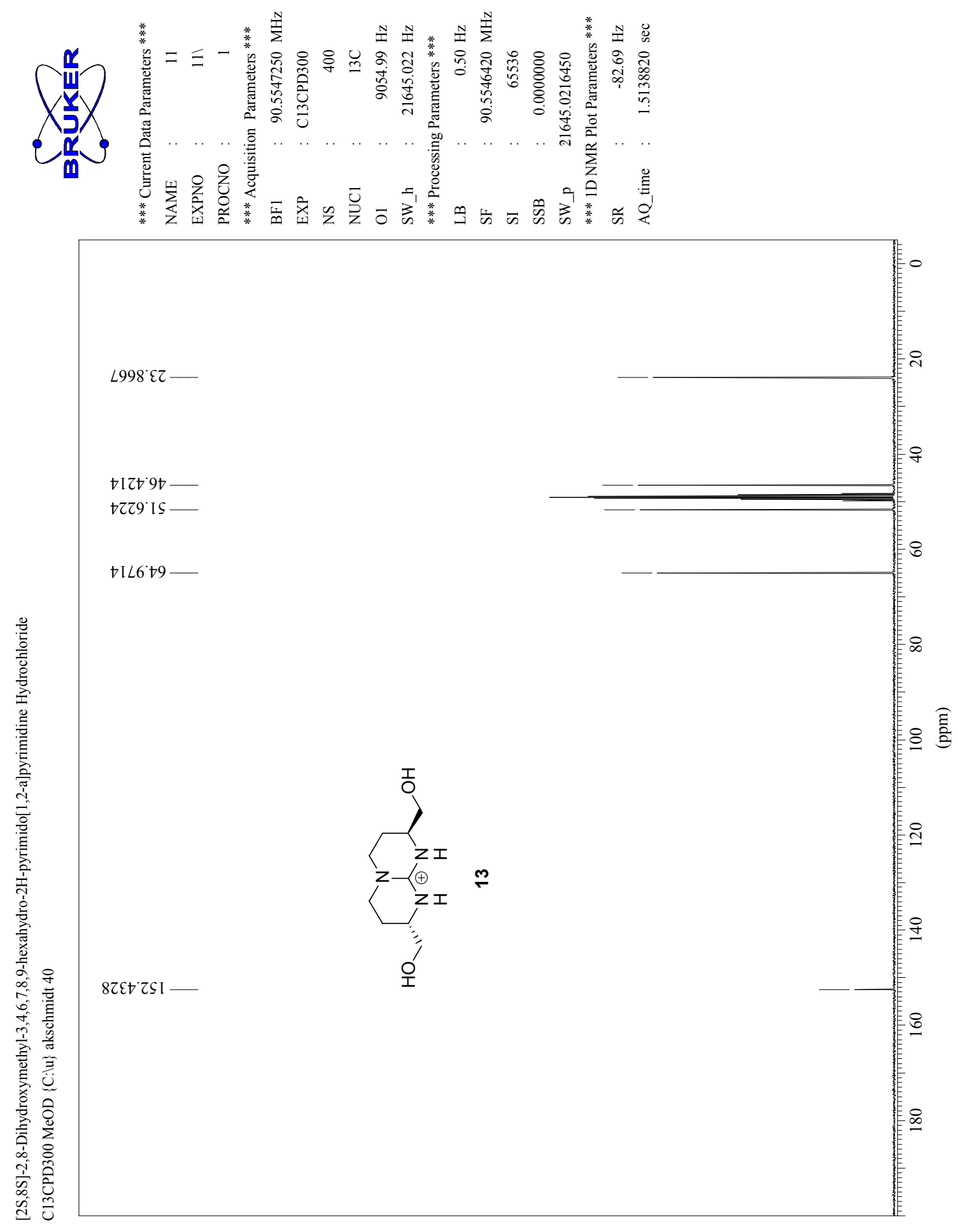

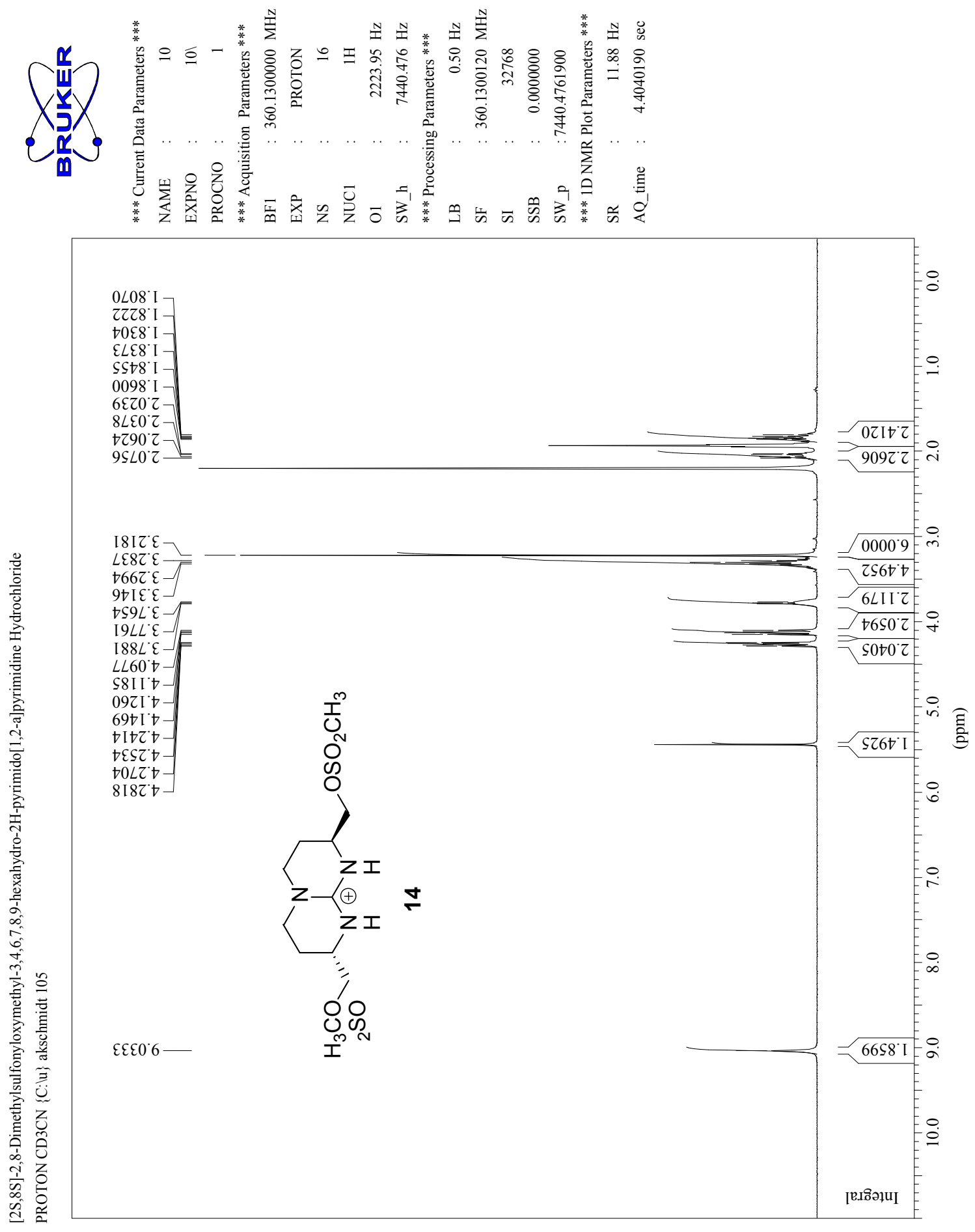

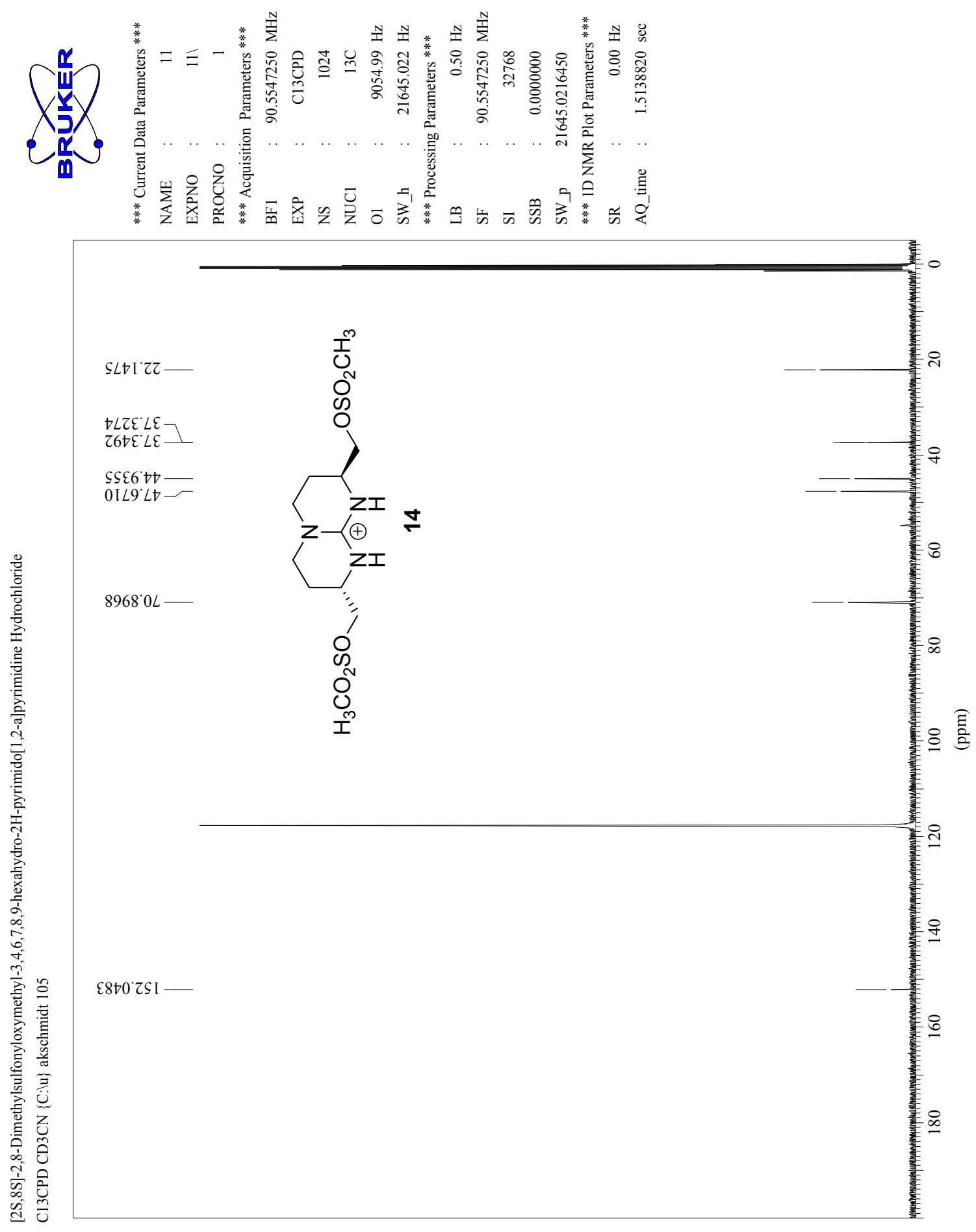

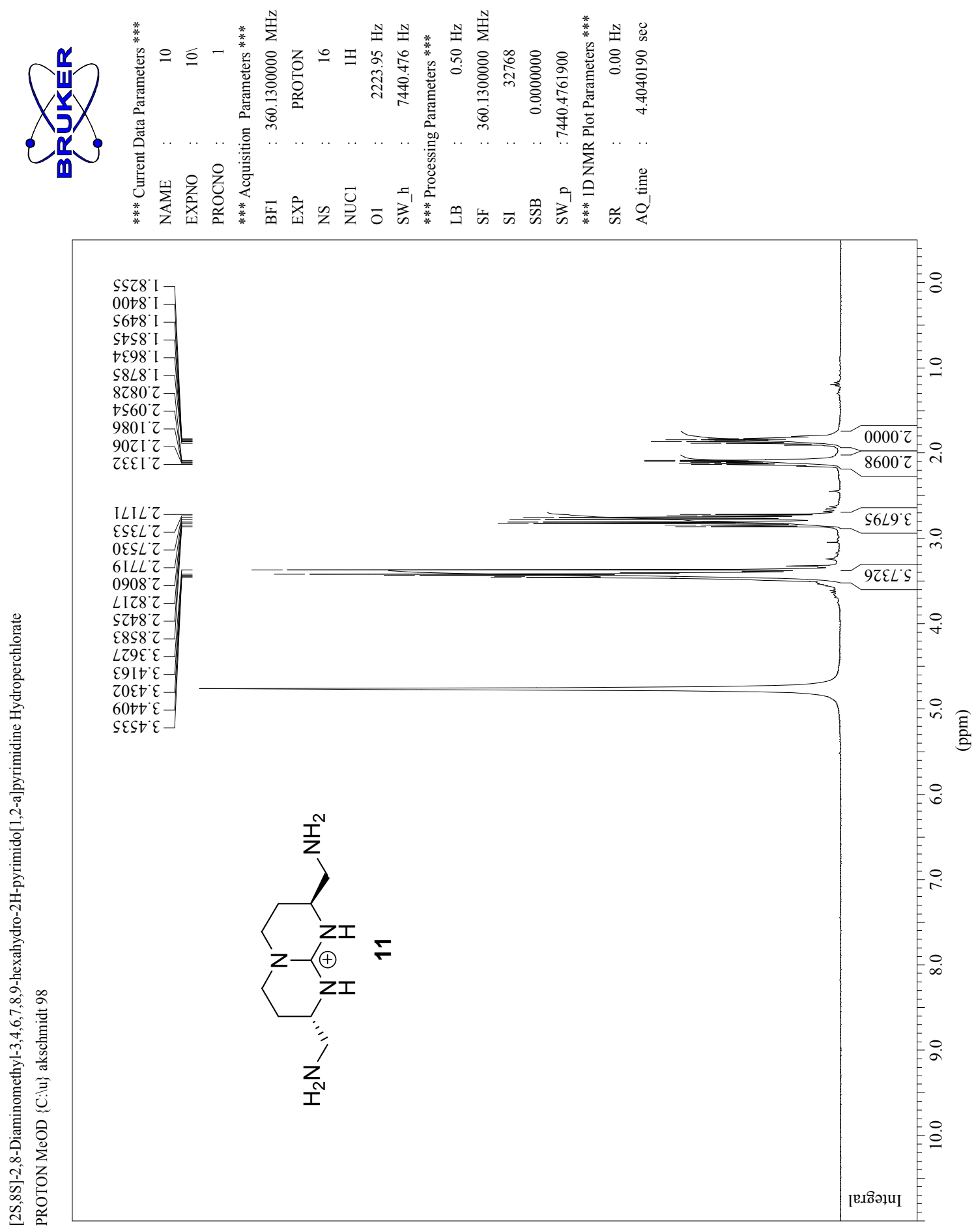

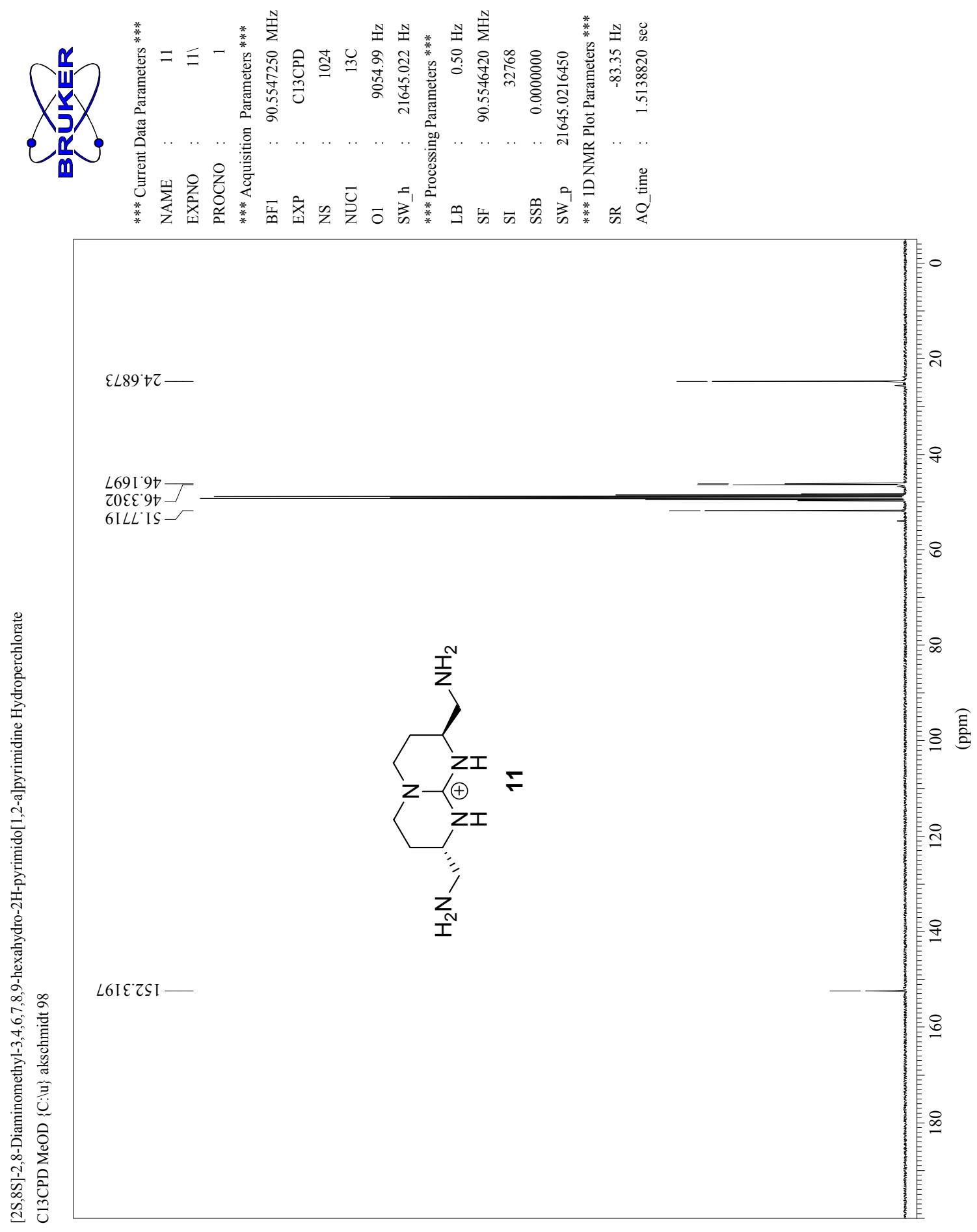

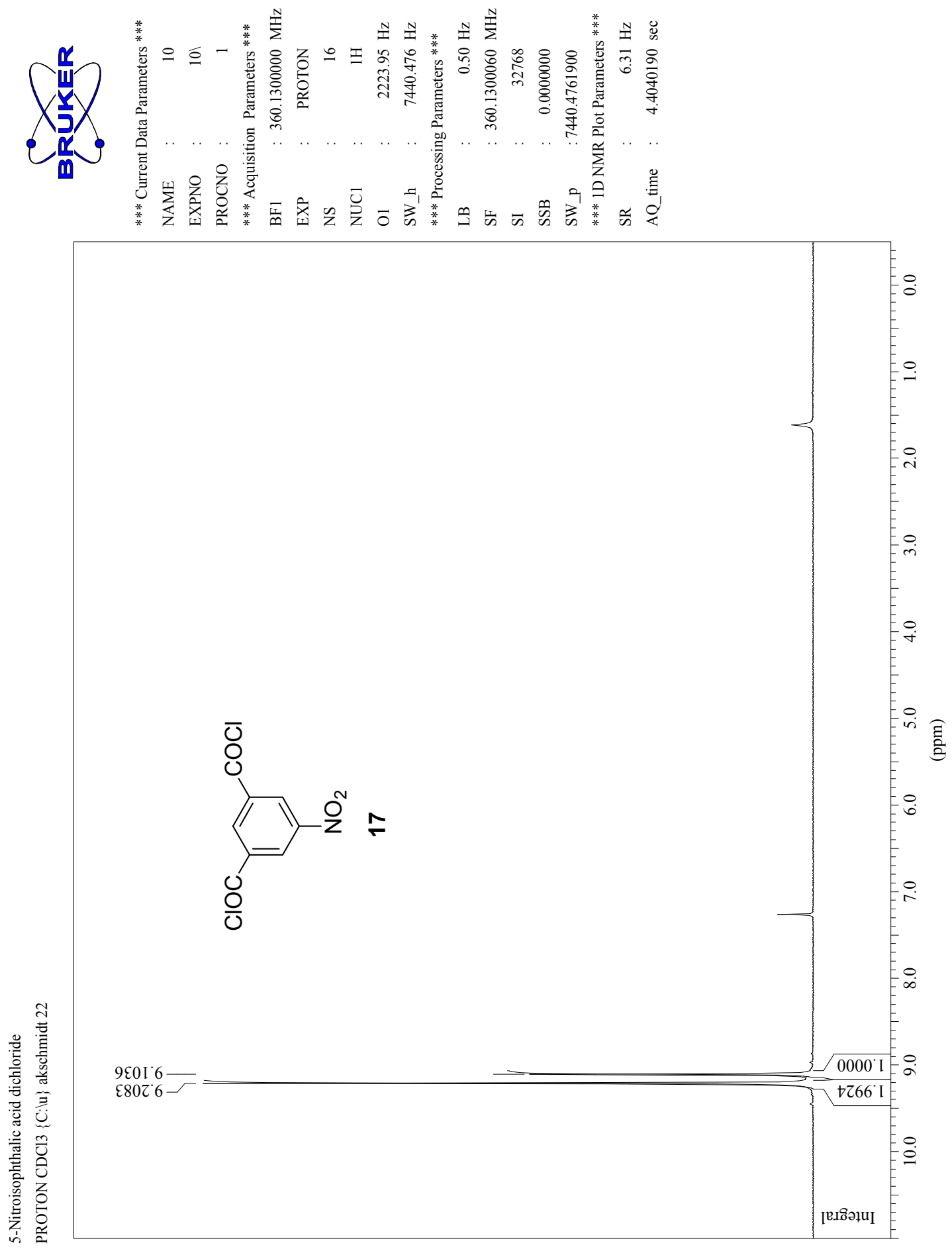

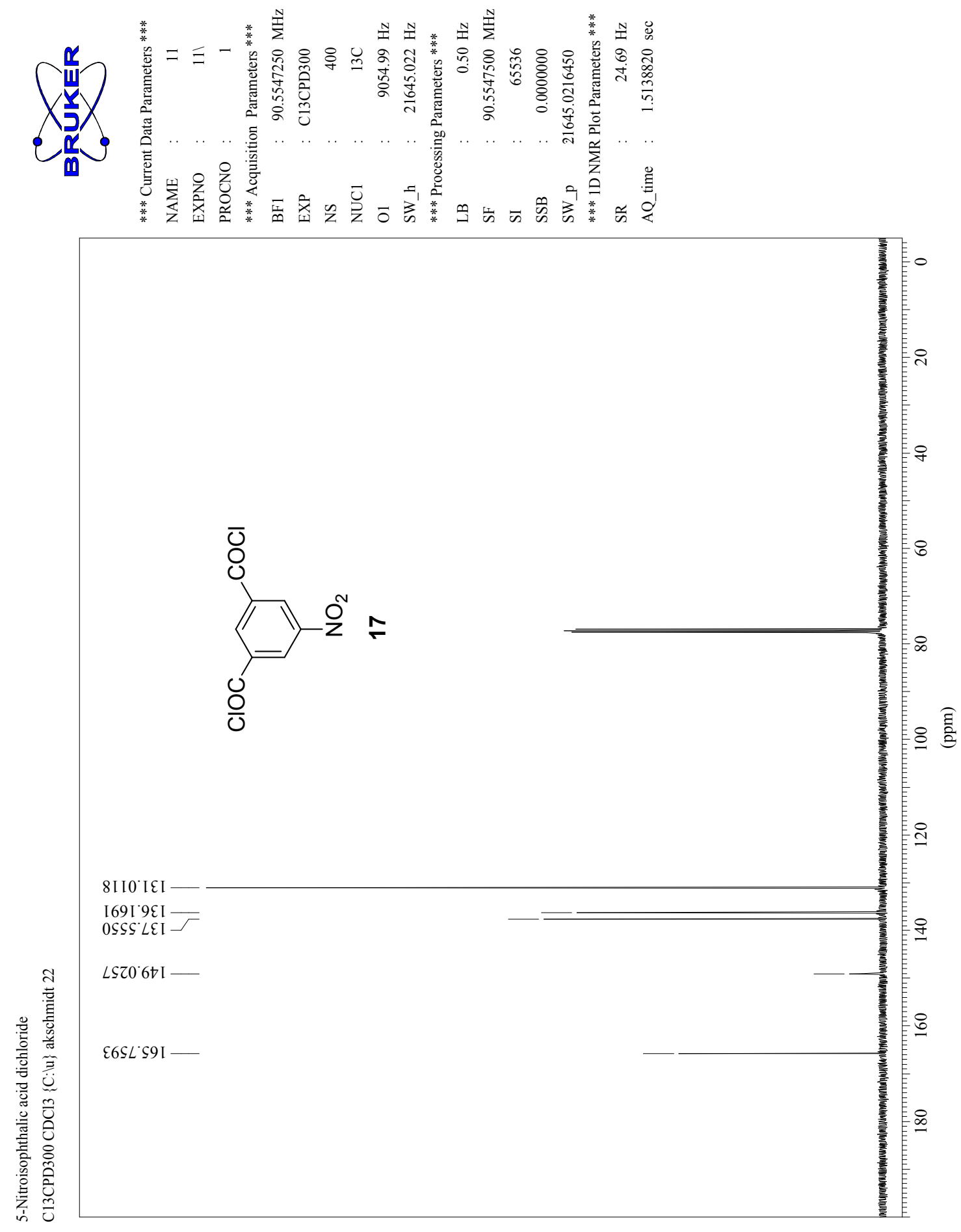

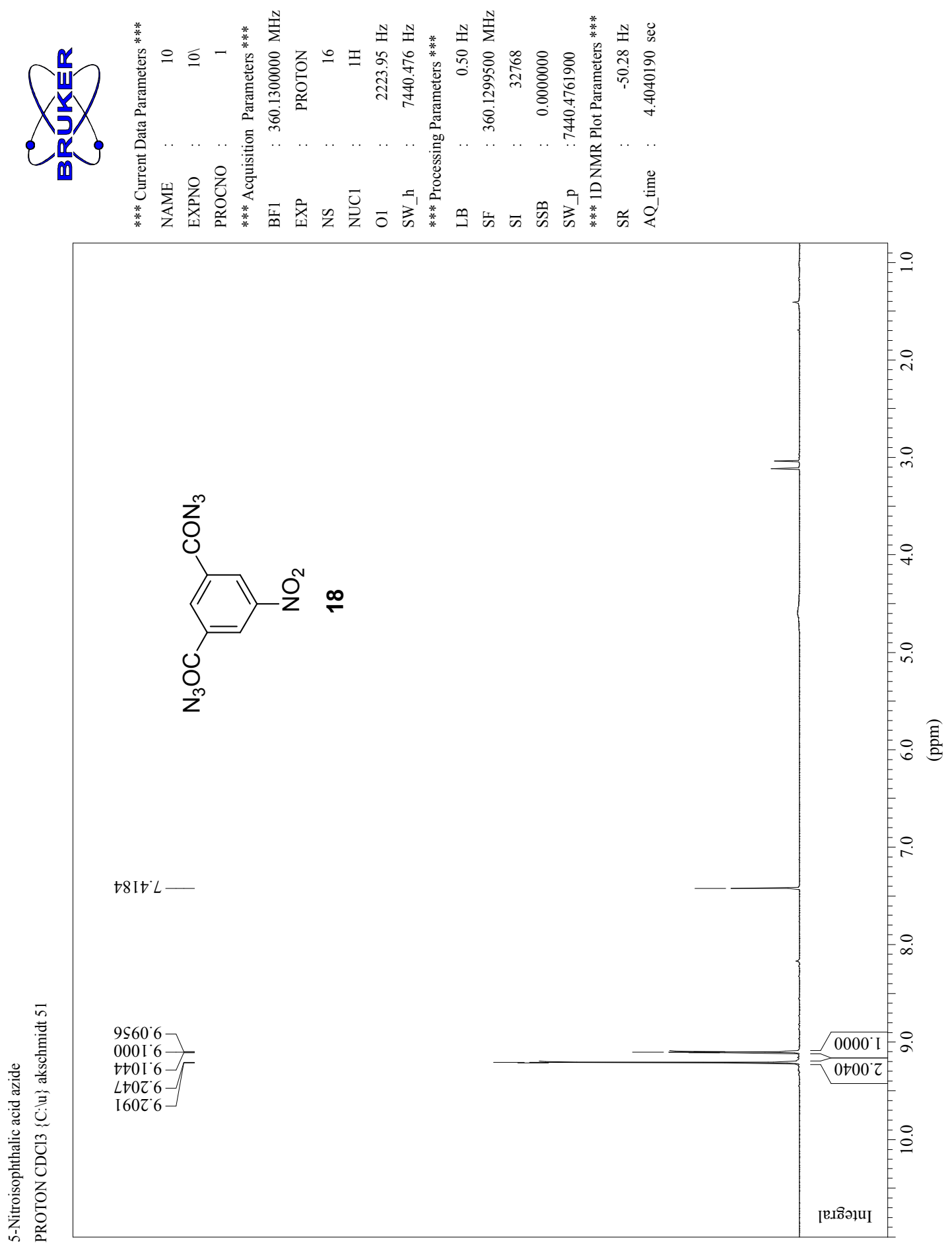

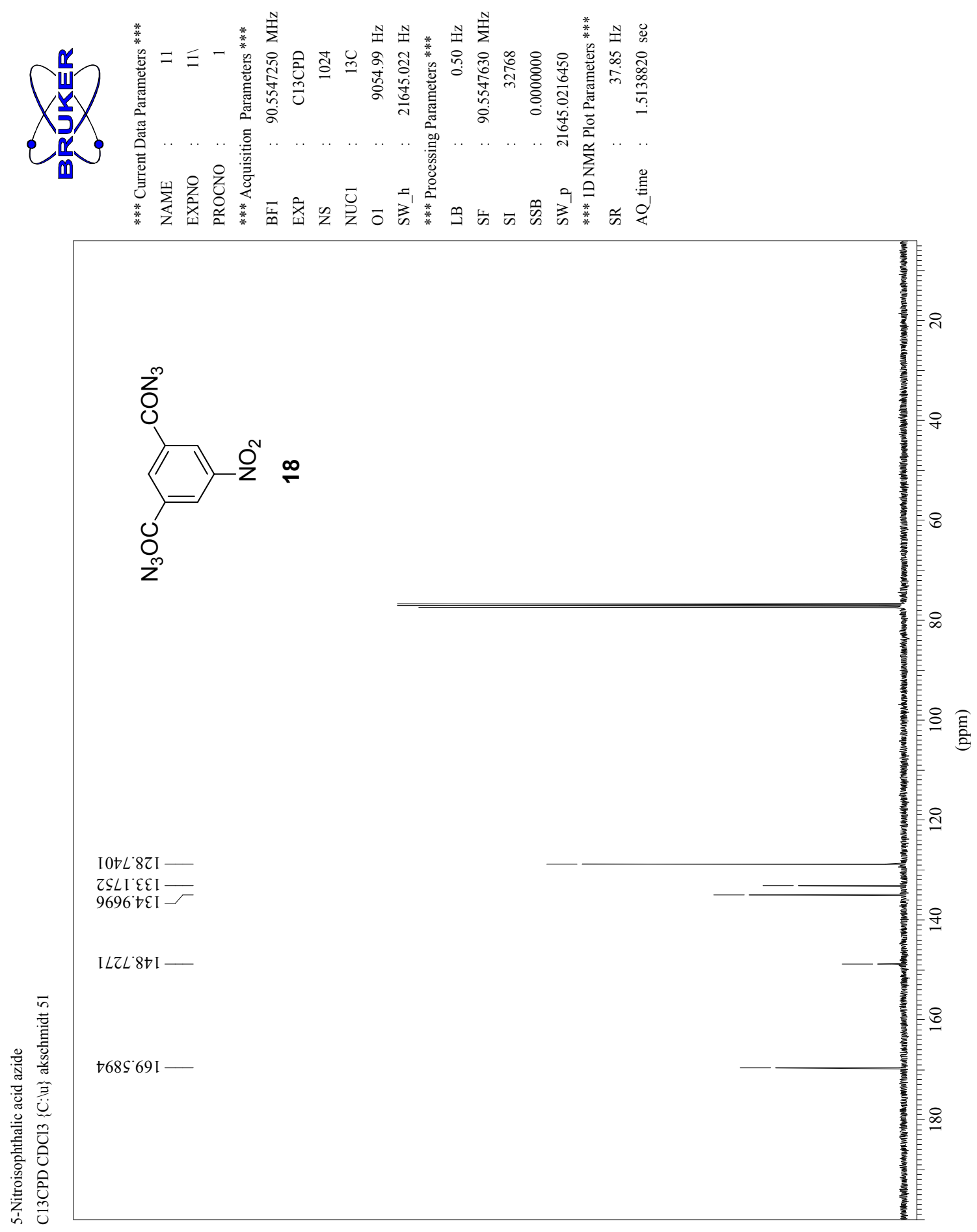

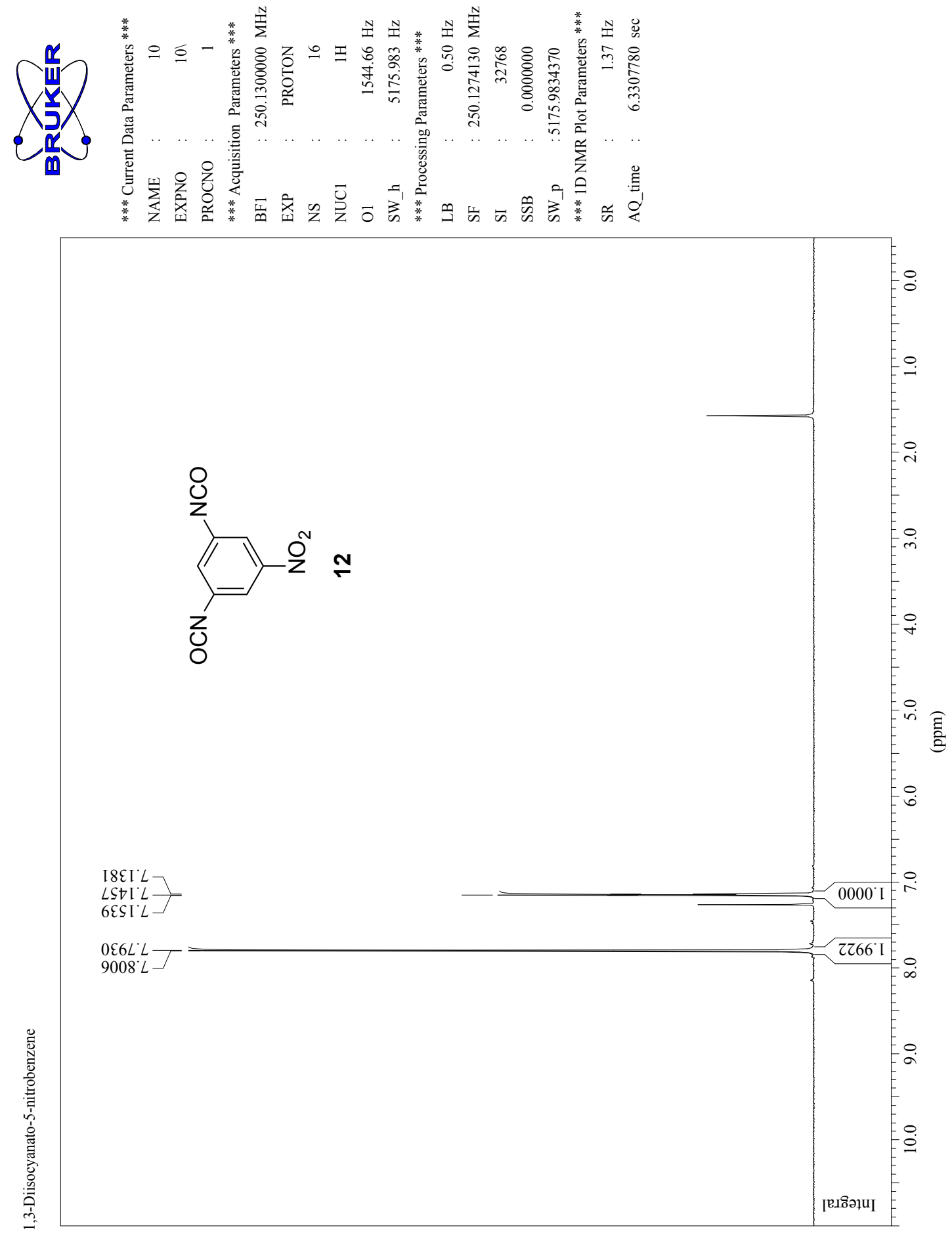

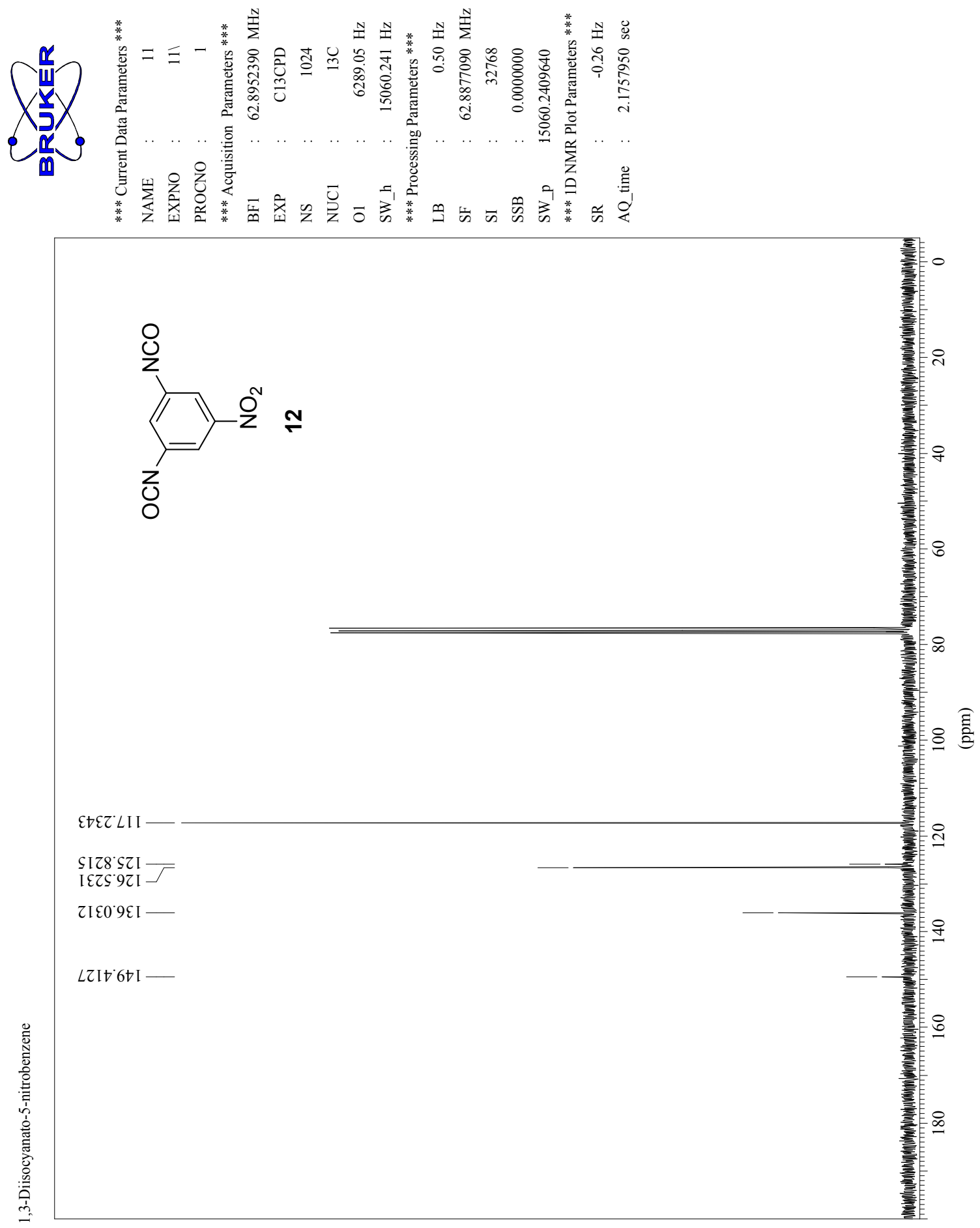

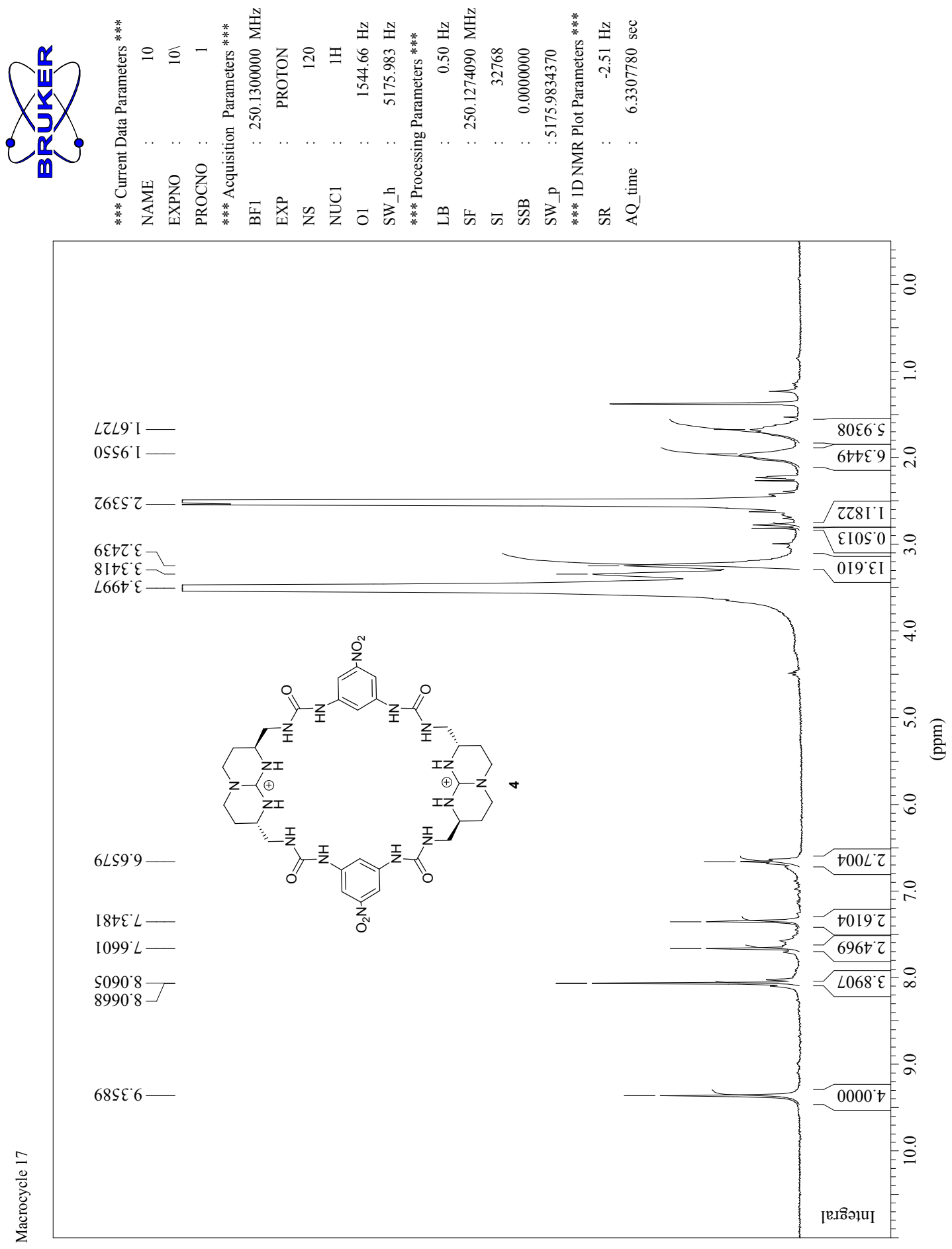

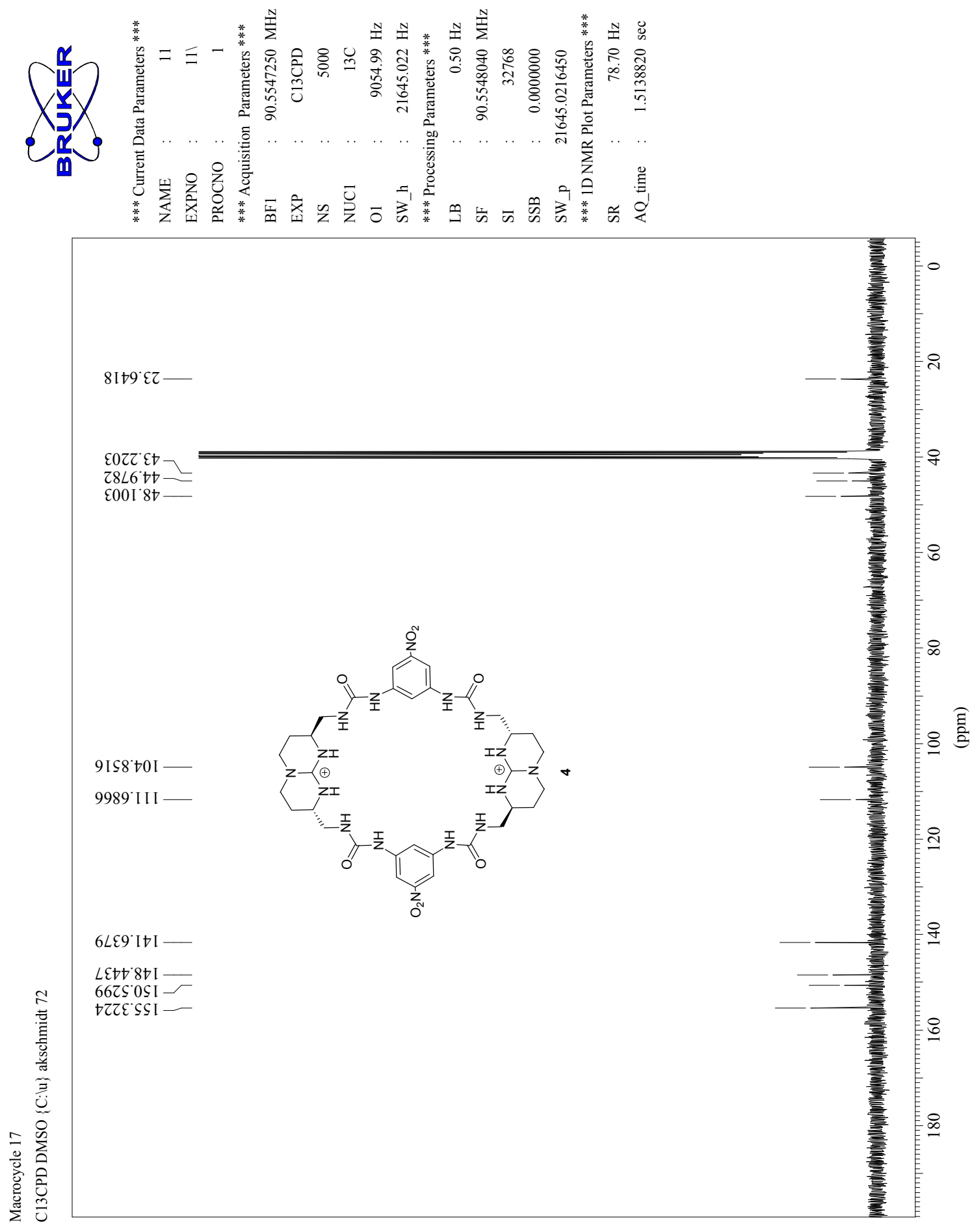

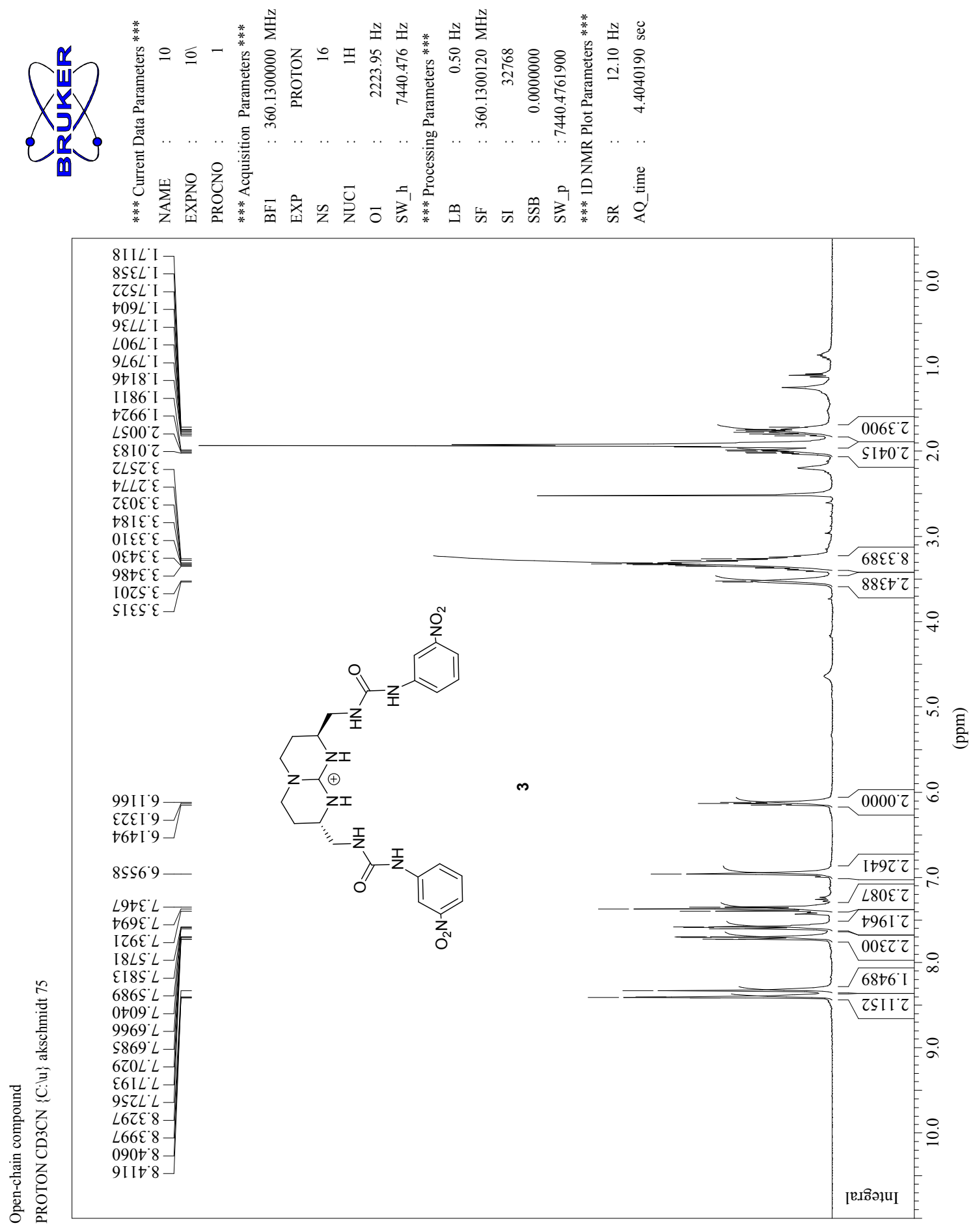


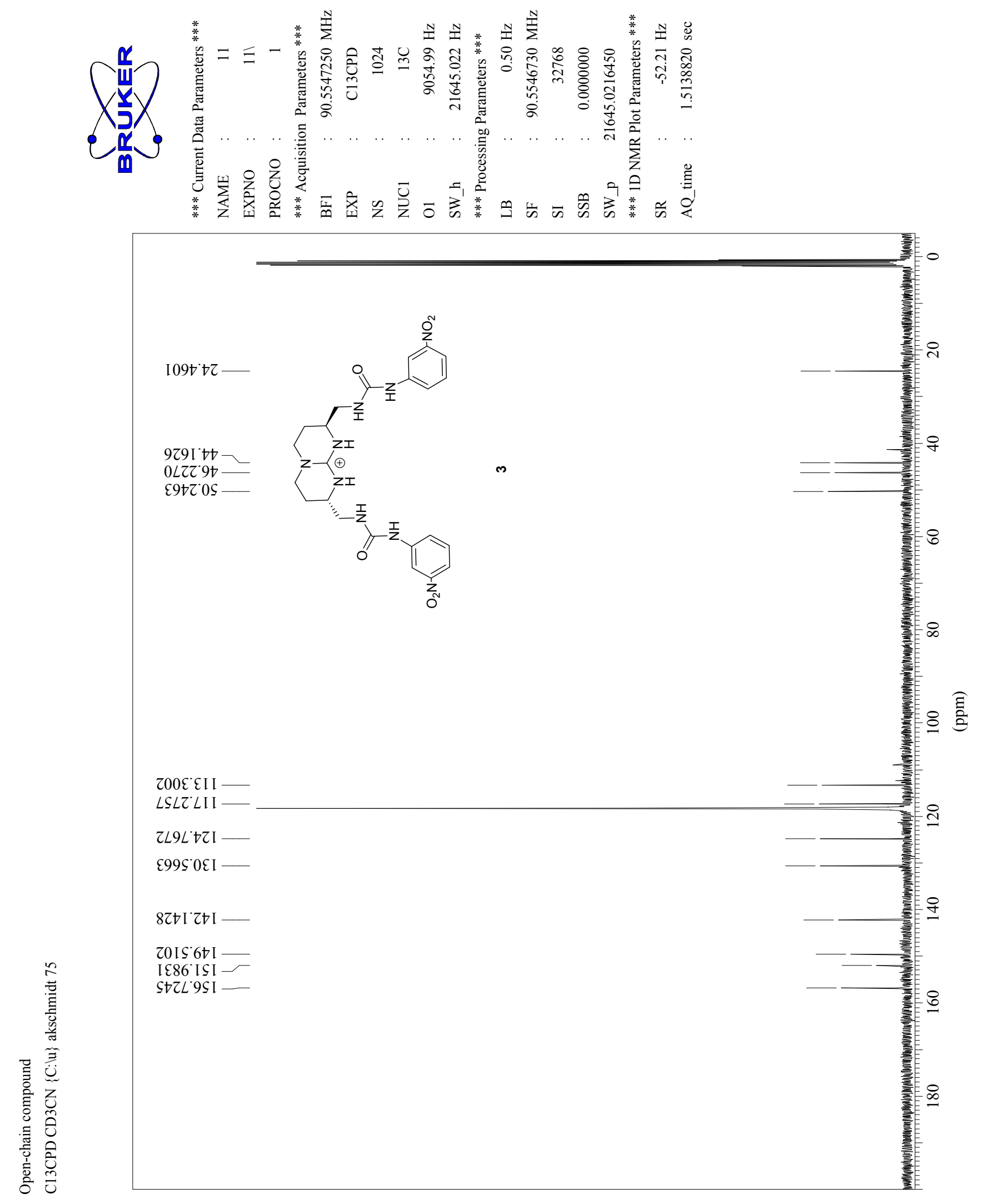


3. Mass spectra (ESI-MS) of the compounds 2-7, 10-11 and HRMS of 2 and 11

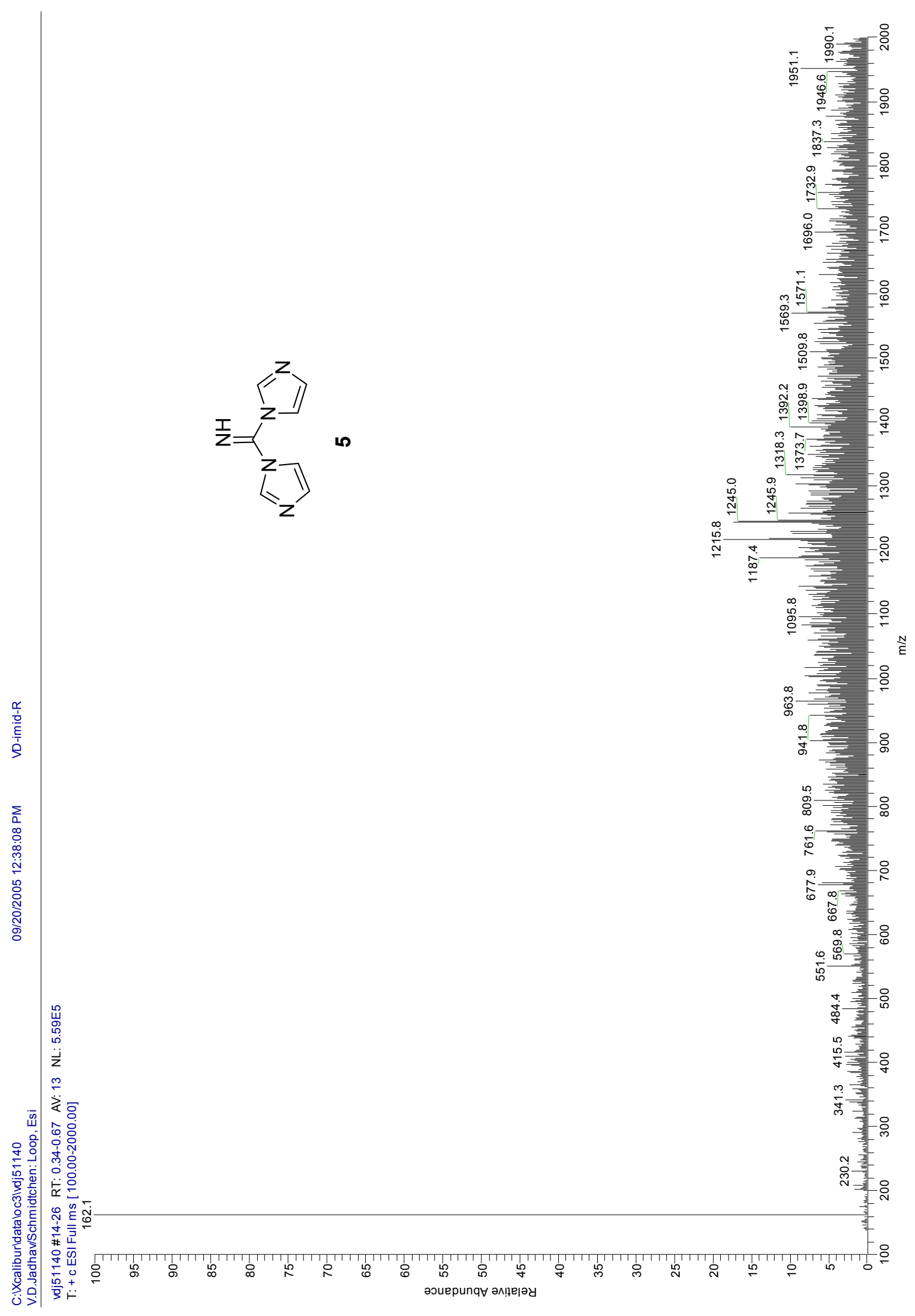




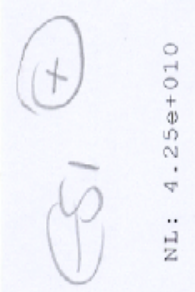
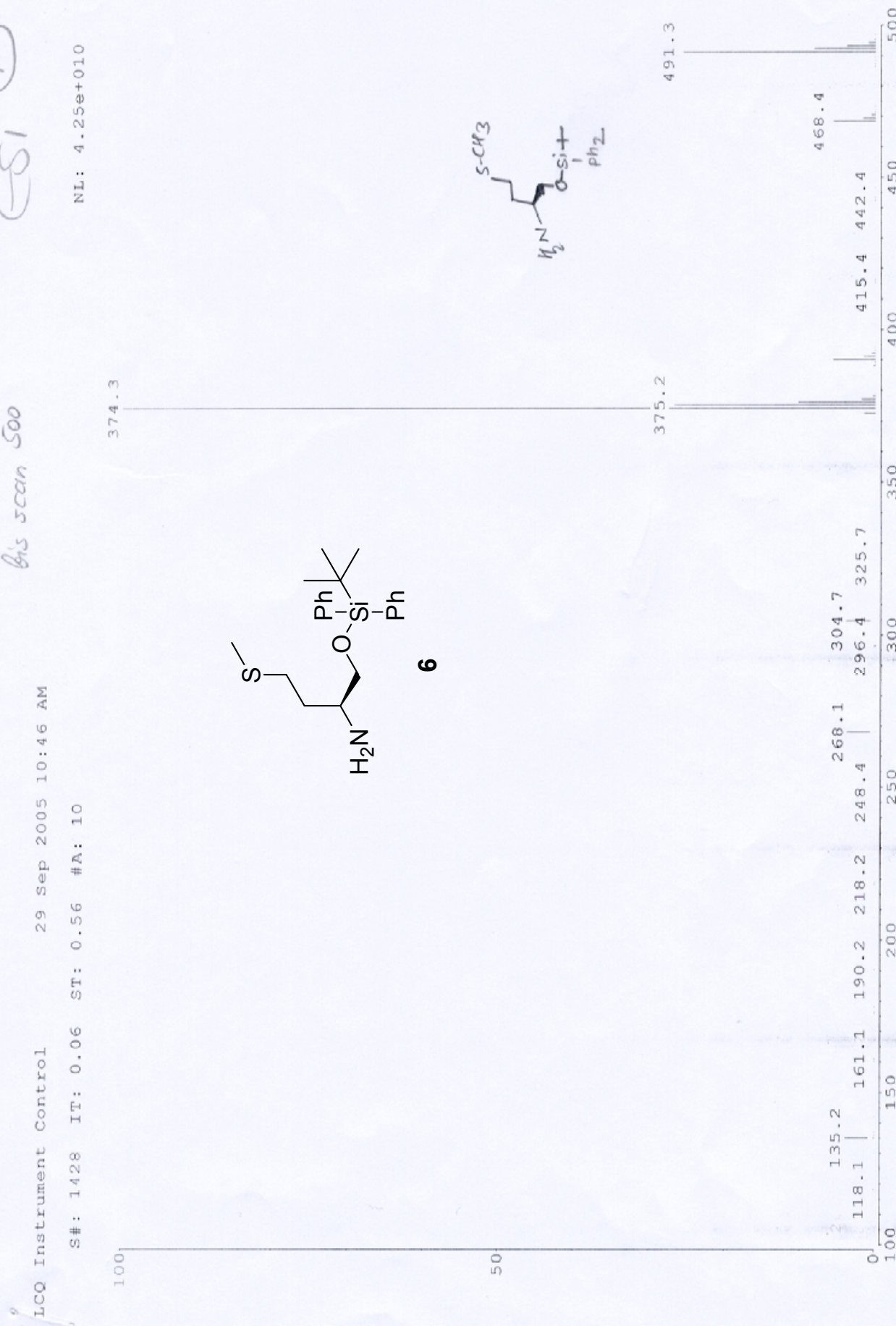

○

$\ddot{\sigma}$

in

$\ddot{4}$

0
$\vdots$
0
$\ddot{H}$
$\ddot{H}$

है

군

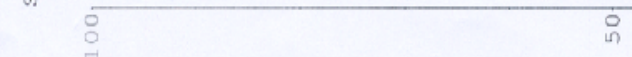




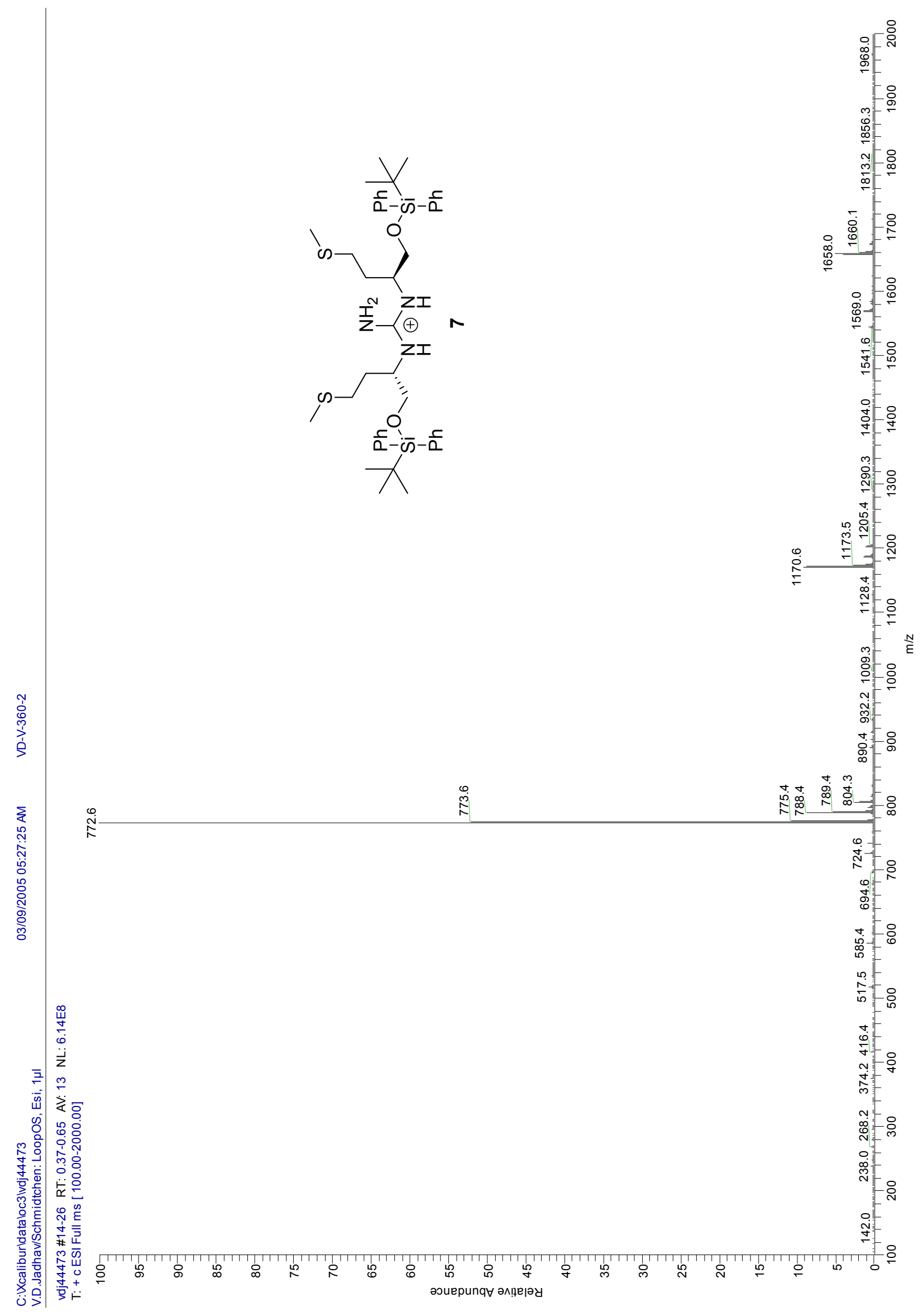




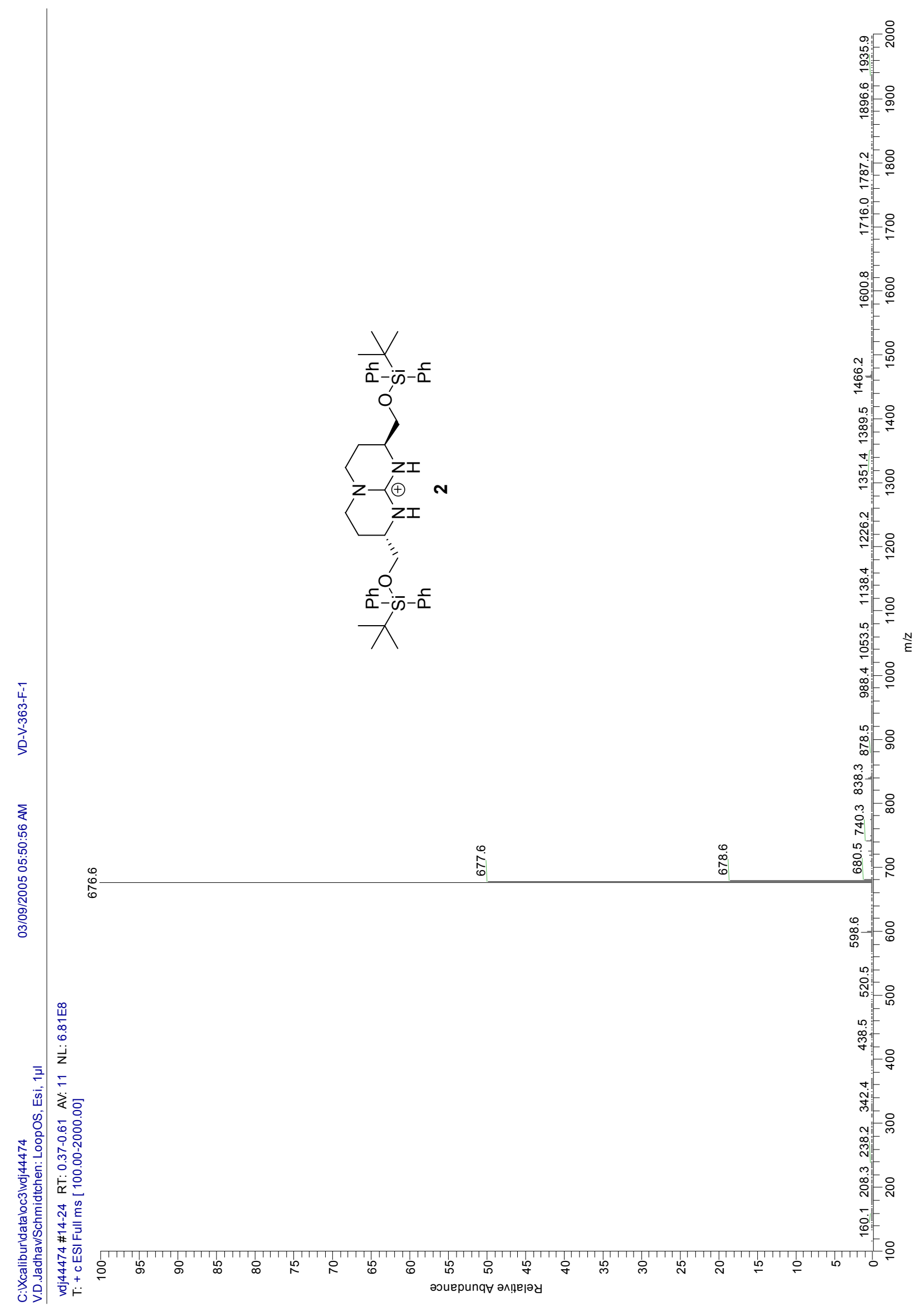




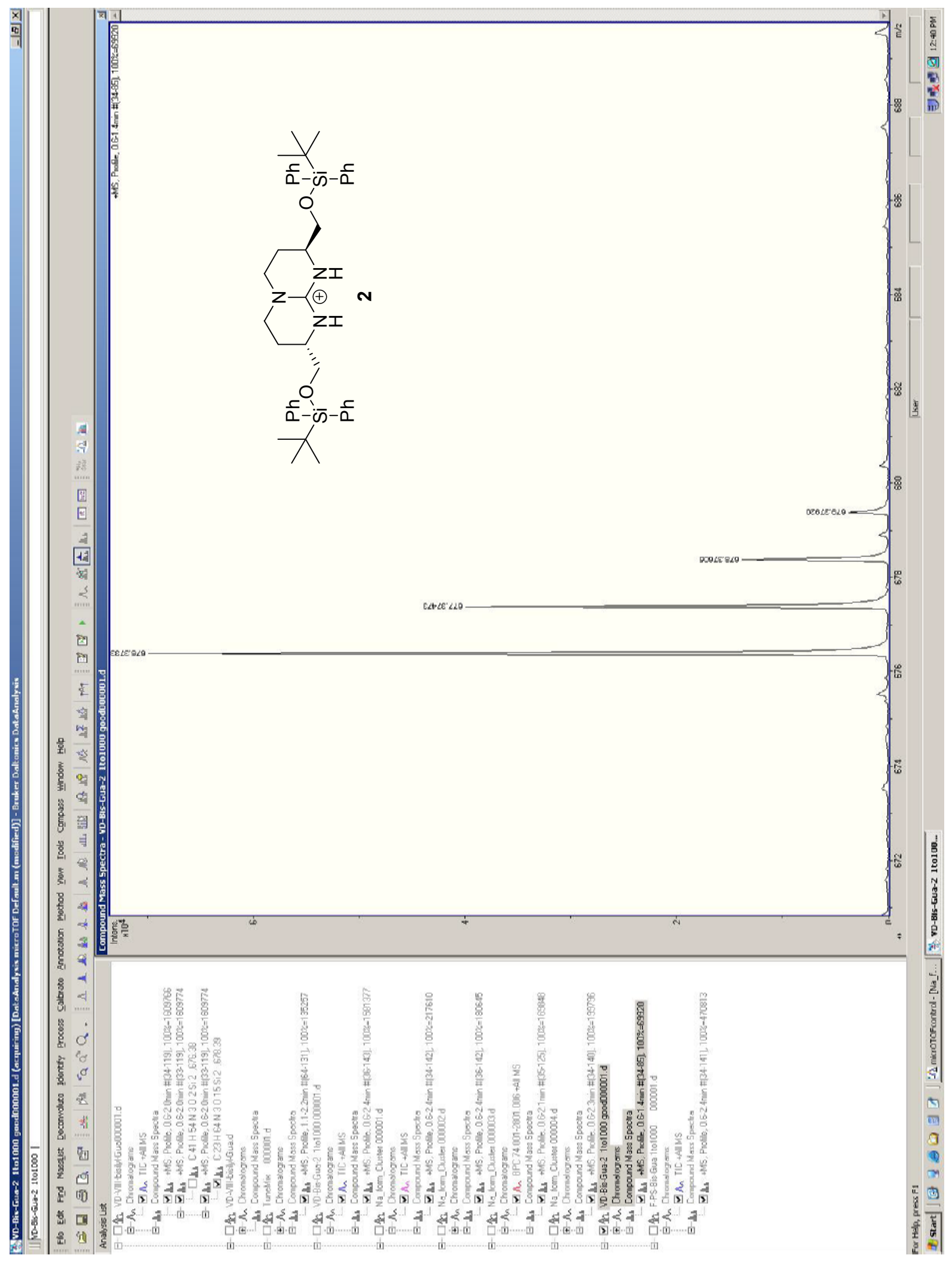




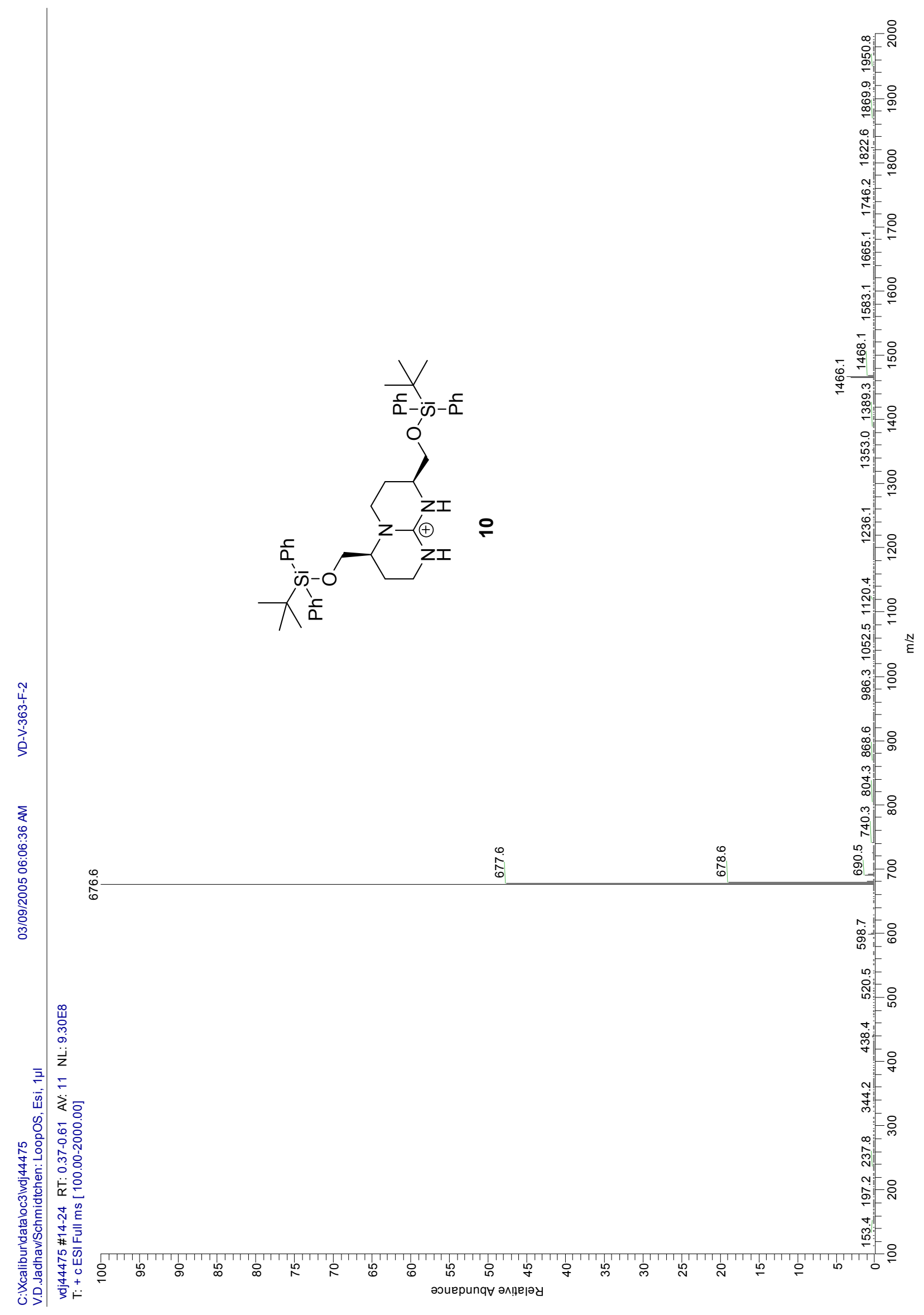




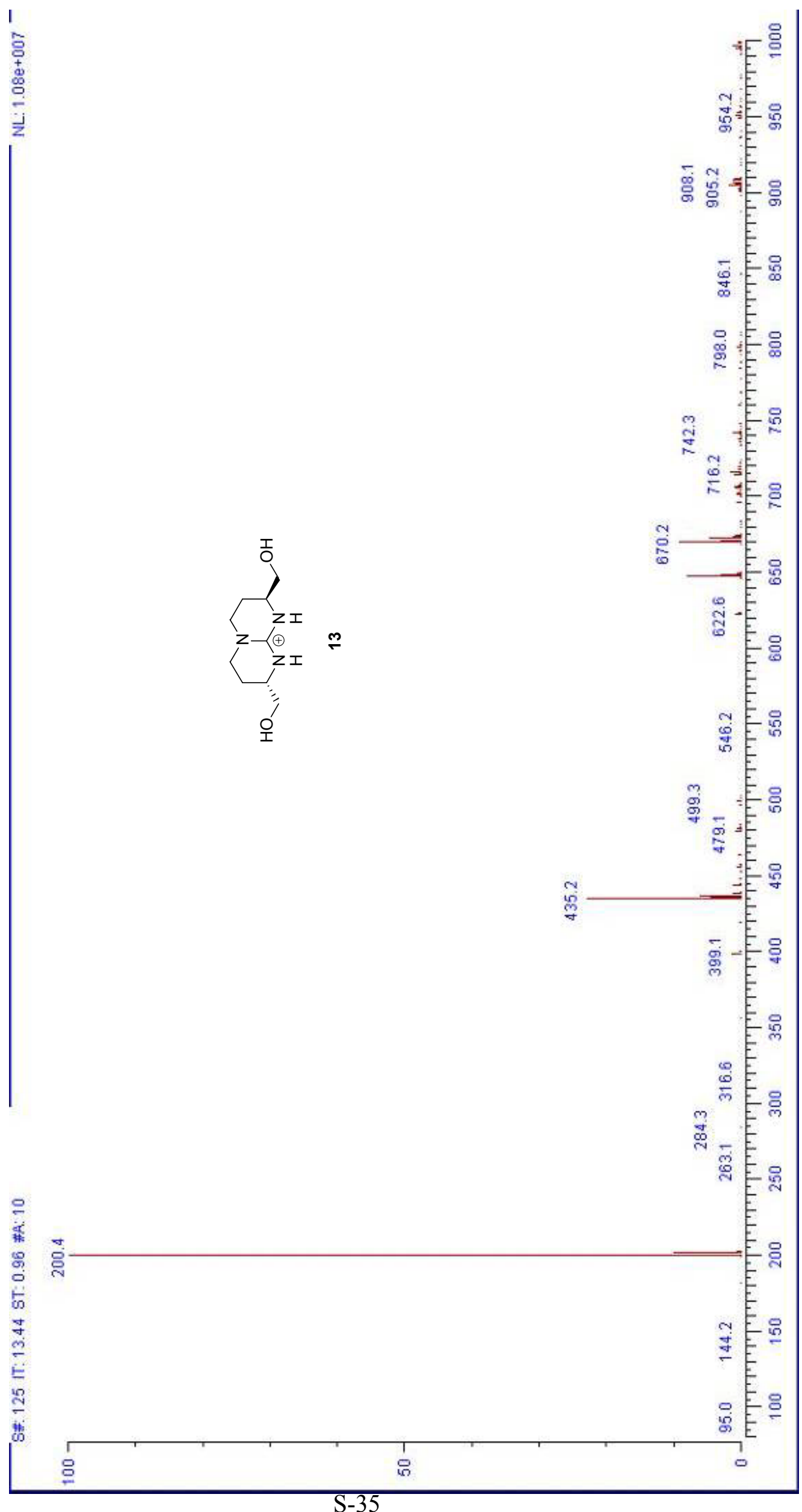




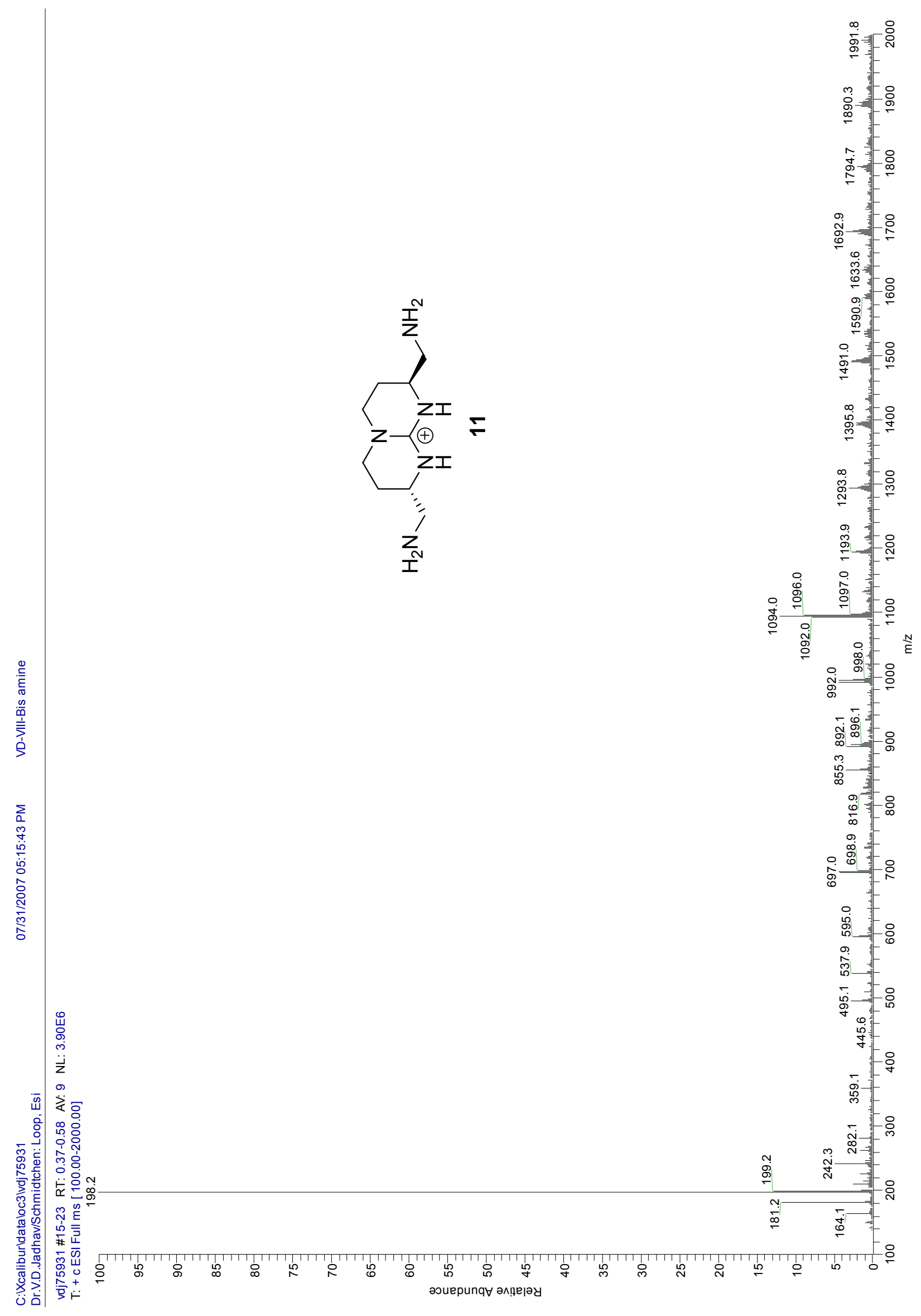




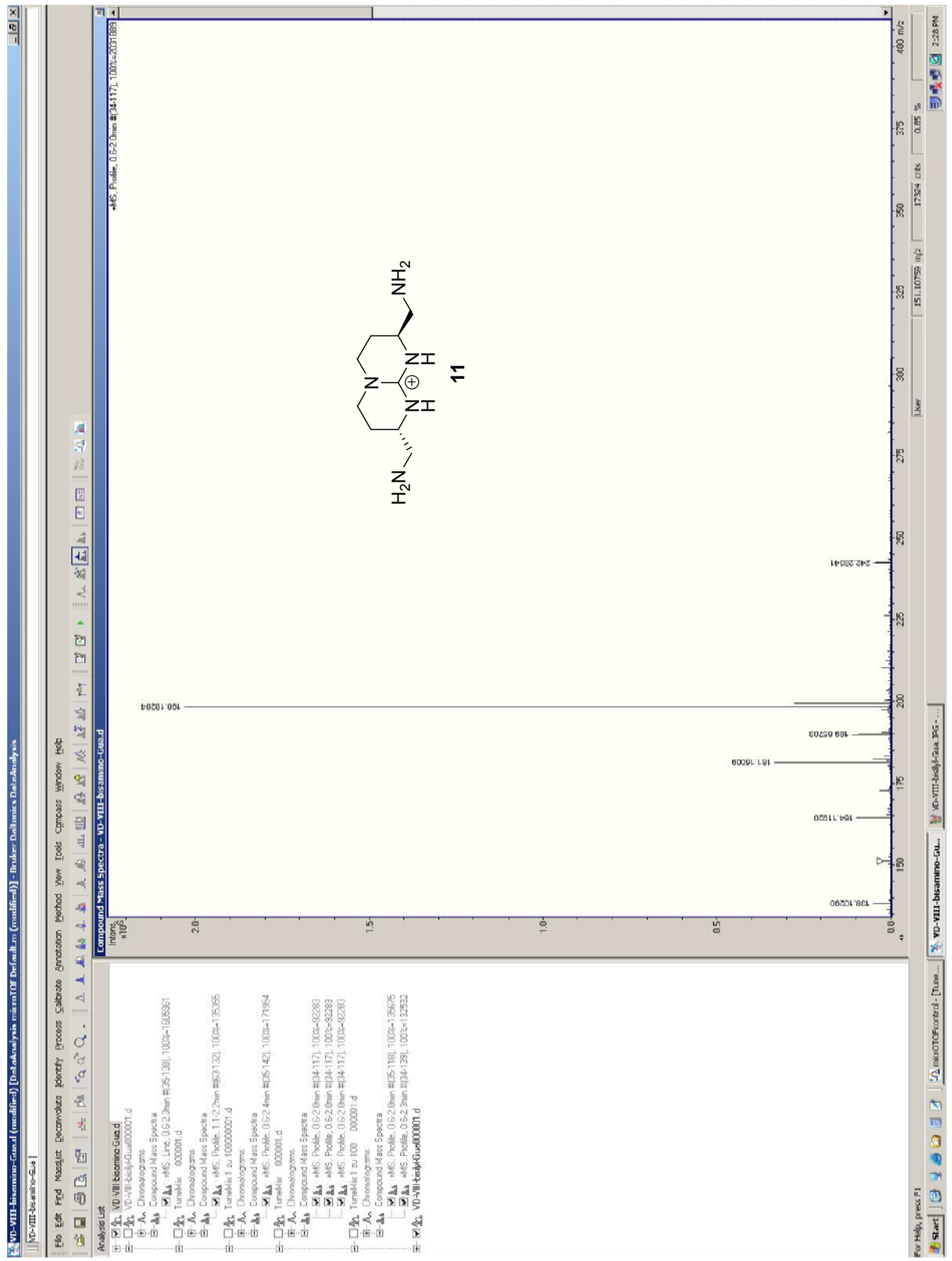




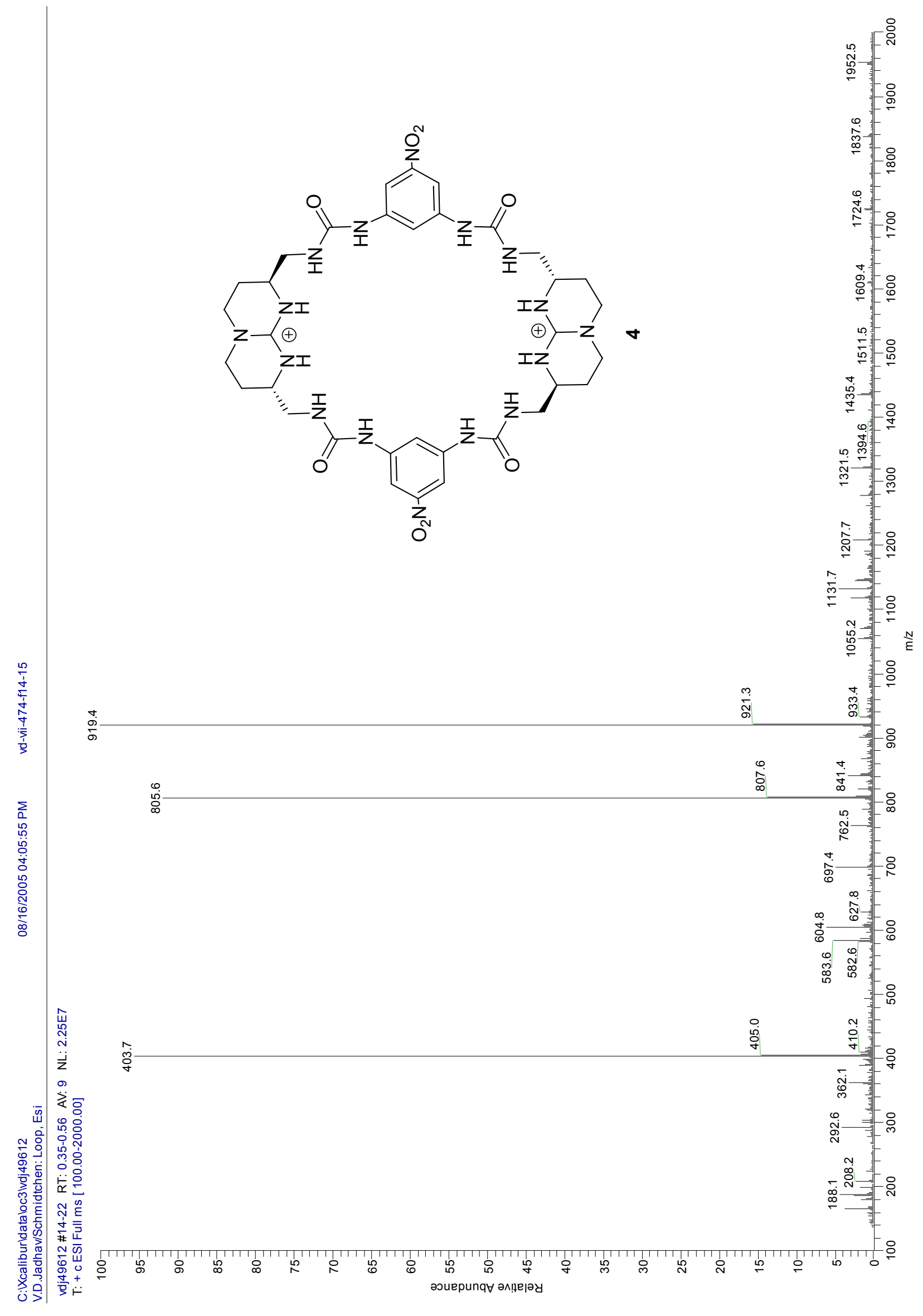




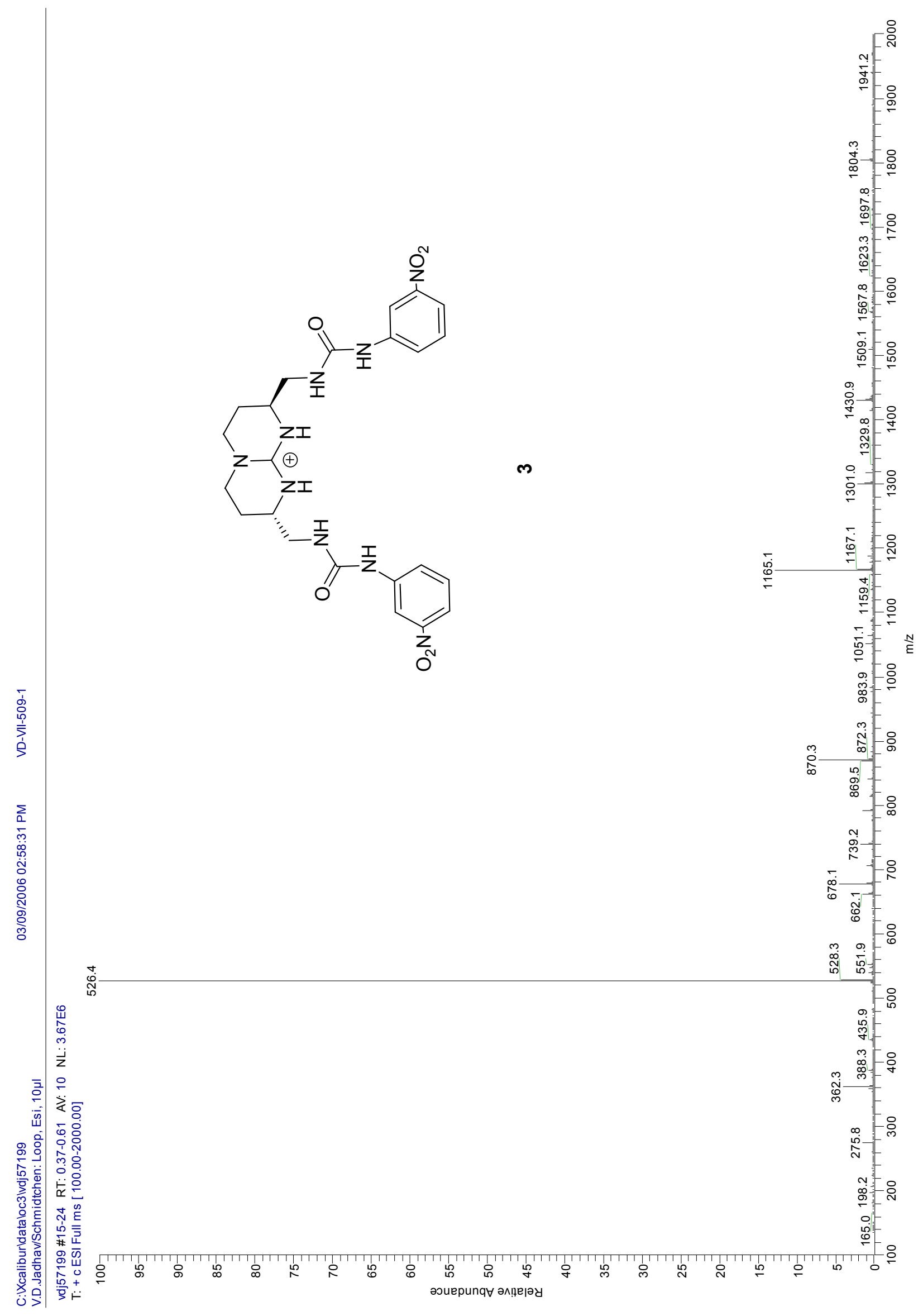




\section{Supporting material for entries depicted in table 1}

a) ITC traces of the titration of $\mathbf{4}$ with terephthalate ${ }^{2-} \mathbf{2 1}$ (left) and PCVC $\mathbf{P C}^{\mathbf{2}} \mathbf{2 0}$ (right). Titration mode: host into guest (tetraethylammonium salt) solution; one-site-model; ligand-in-cell
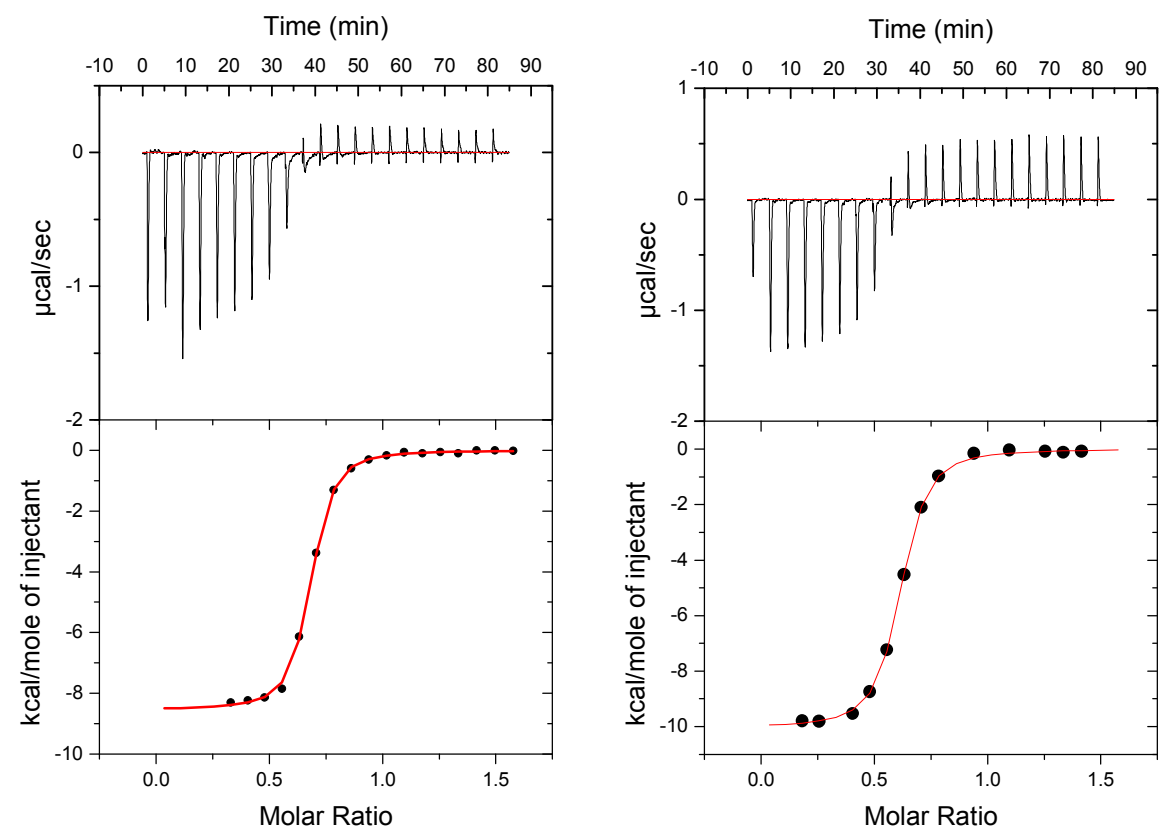

b) ITC traces of the titration of 4 with isophthalate ${ }^{2-} \mathbf{2 0}$ (left) and phthalate ${ }^{2-} \mathbf{1 9}$ (right). Titration mode: host into guest (tetraethylammonium salt) solution; one-site-model; ligand-in-cell
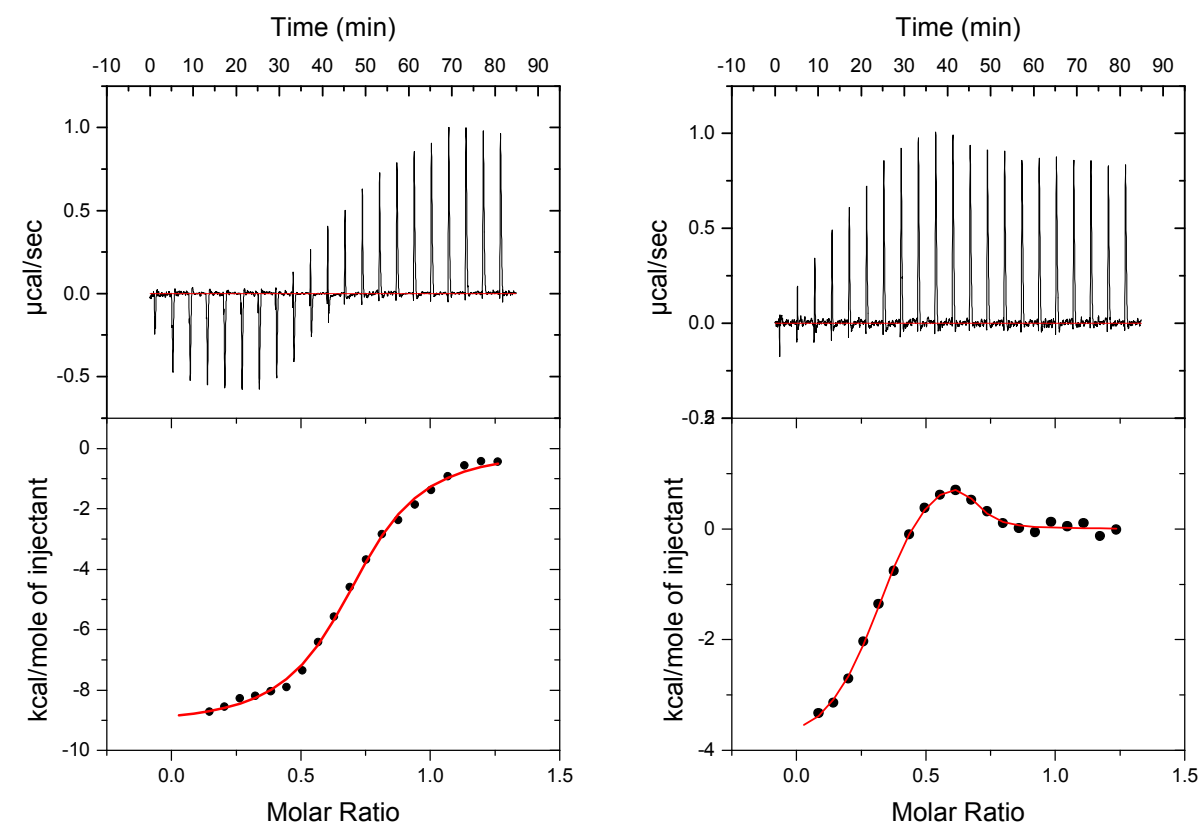
c) ITC traces of the titration of macrocycle 4 with hydrogen phosphate p $^{2-}$ (left) and naphthalene dicarboxylate 22 (right). Titration mode: host into guest (tetraethylammonium salt) solution; one-site-model; ligand-in-cell
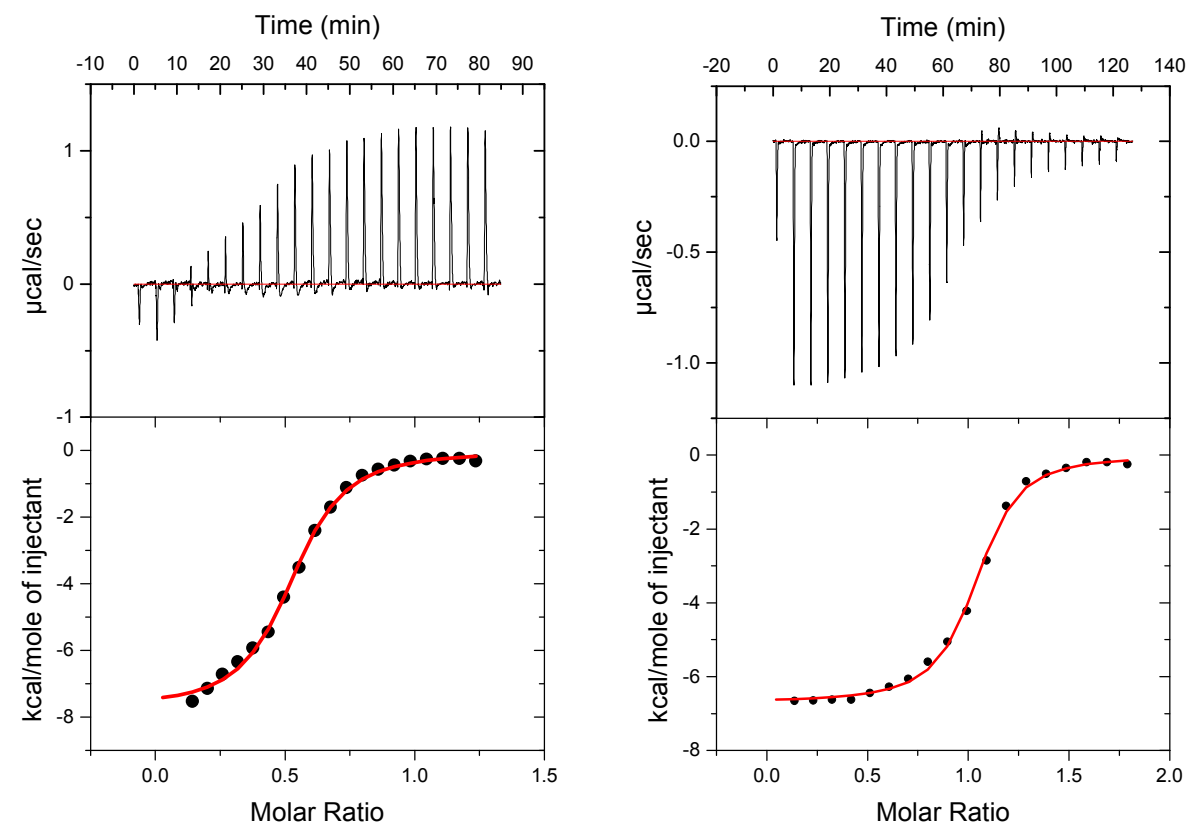

d) ITC traces of the titration of macrocycle 4 with squarate ${ }^{2-} \mathbf{3 0}$ (left) and oxalate s- $^{25}$ (right).

Titration mode: host into guest (tetraethylammonium salt) solution; one-site-model; ligand-in-cell
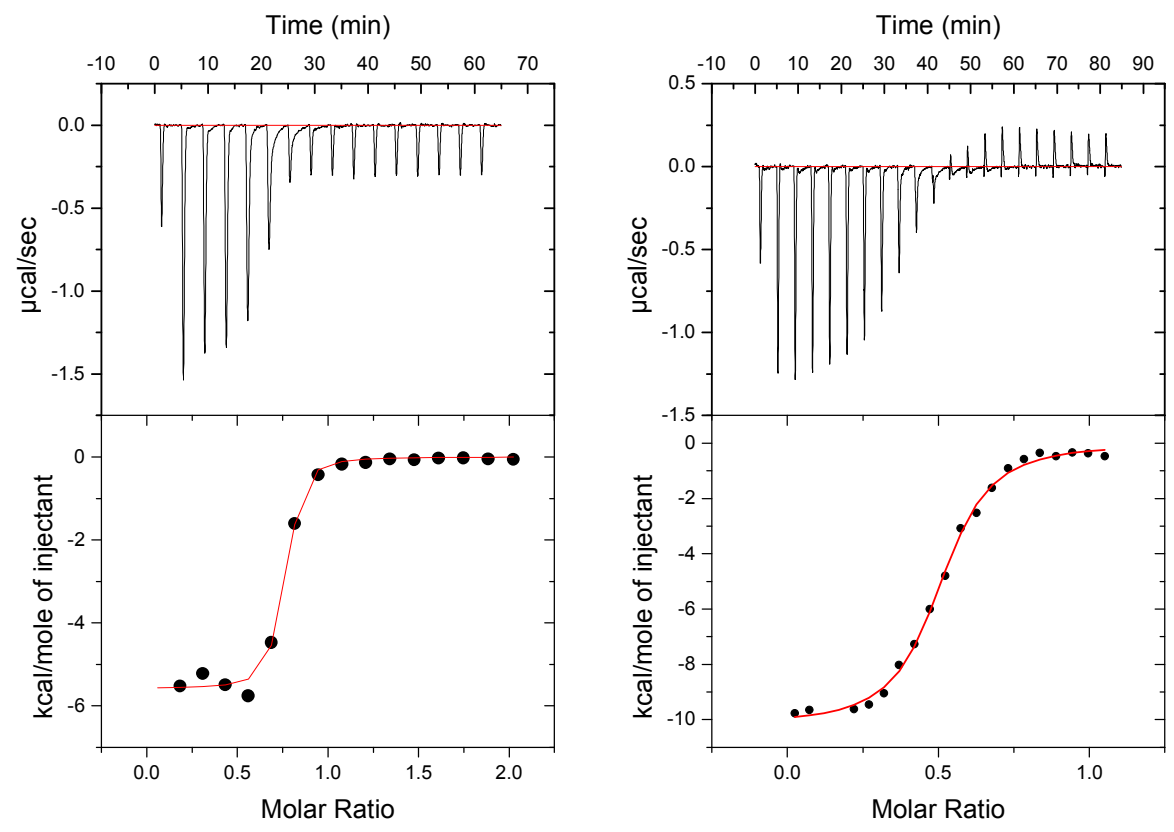
e) ITC traces of the titration of macrocycle 4 with succinate ${ }^{2-} \mathbf{2 6}$ (left), titration mode: guest (tetraethylammonium salt) into host solution; one-site-model and with fumarate ${ }^{2-} \mathbf{2 7}$ (right), titration mode: host into guest (tetraethylammonium salt) solution; 2 sequential site model; ligand-in-cell
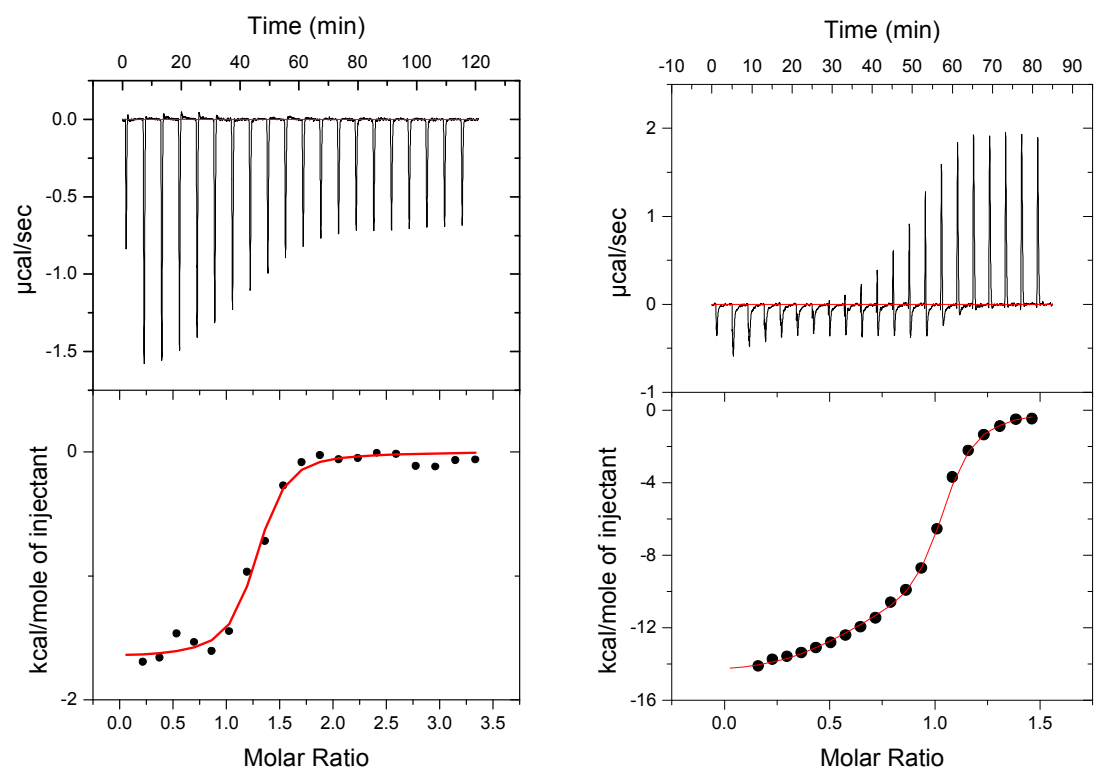

f) ITC traces of the titration of macrocycle 4 with glutaconate ${ }^{2-} \mathbf{2 8}$ (left), titration mode: host into guest (tetraethylammonium salt) solution; 2 sequential site model; ligand-in-cell and with t,tmuconate $^{2-} 29$ (right), titration mode: host into guest (tetraethylammonium salt) solution; one-sitemodel; ligand-in-cell.
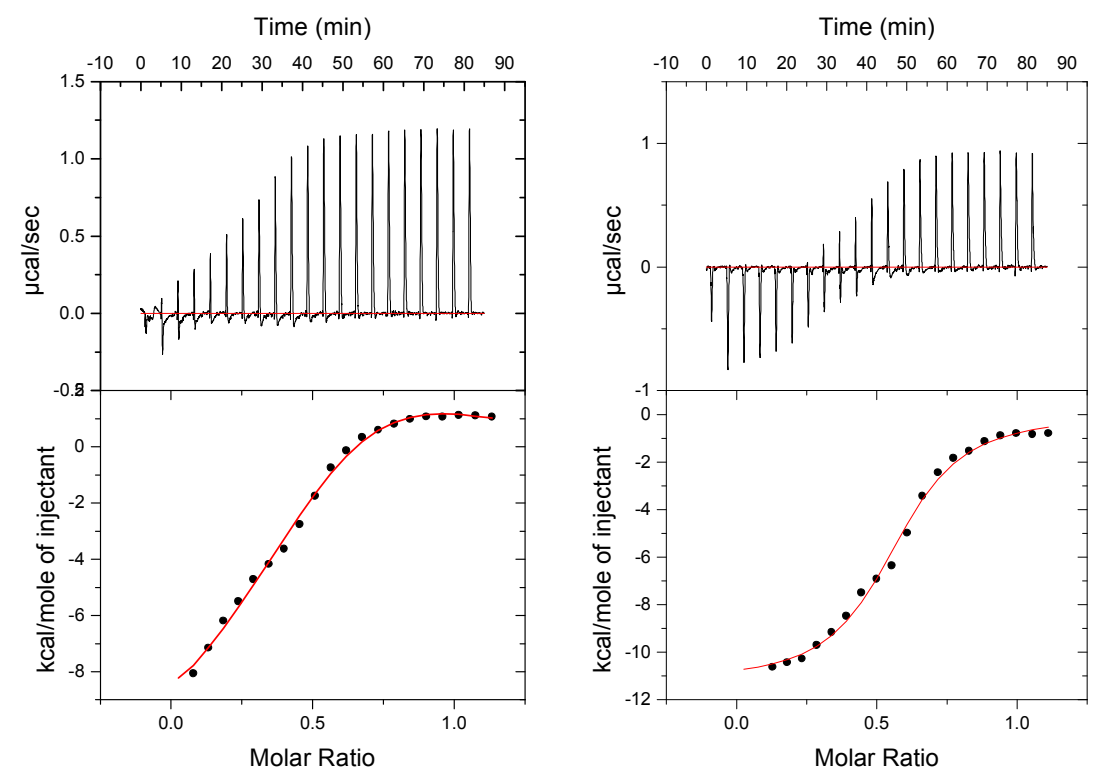


\section{Supporting material for entries depicted in table 2}

a) ITC traces of the titration of macrocycle 4 with $(S, S)-\operatorname{tartrate}^{2-} 31$ (left) and $(\boldsymbol{R}, \boldsymbol{R})-\operatorname{tartrate}^{2-} 32$ (right). Titration mode: host into guest (tetraethylammonium salt) solution; one-site-model; ligand-incell
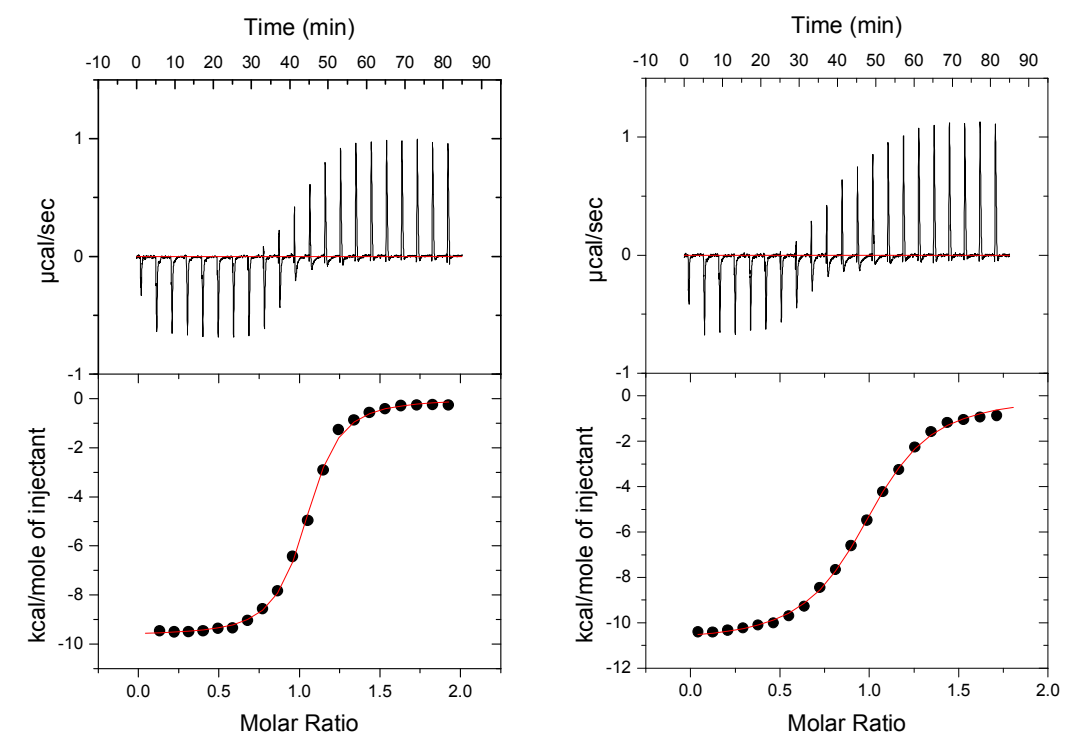

b) ITC traces of the titration of macrocycle 4 with $\boldsymbol{R}$-aspartate ${ }^{1-} 33$ (left) and $S$-aspartate $\mathbf{a r}^{1-} 34$ (right). Titration mode: host into guest (tetraethylammonium salt) solution; one-site-model; ligand-incell
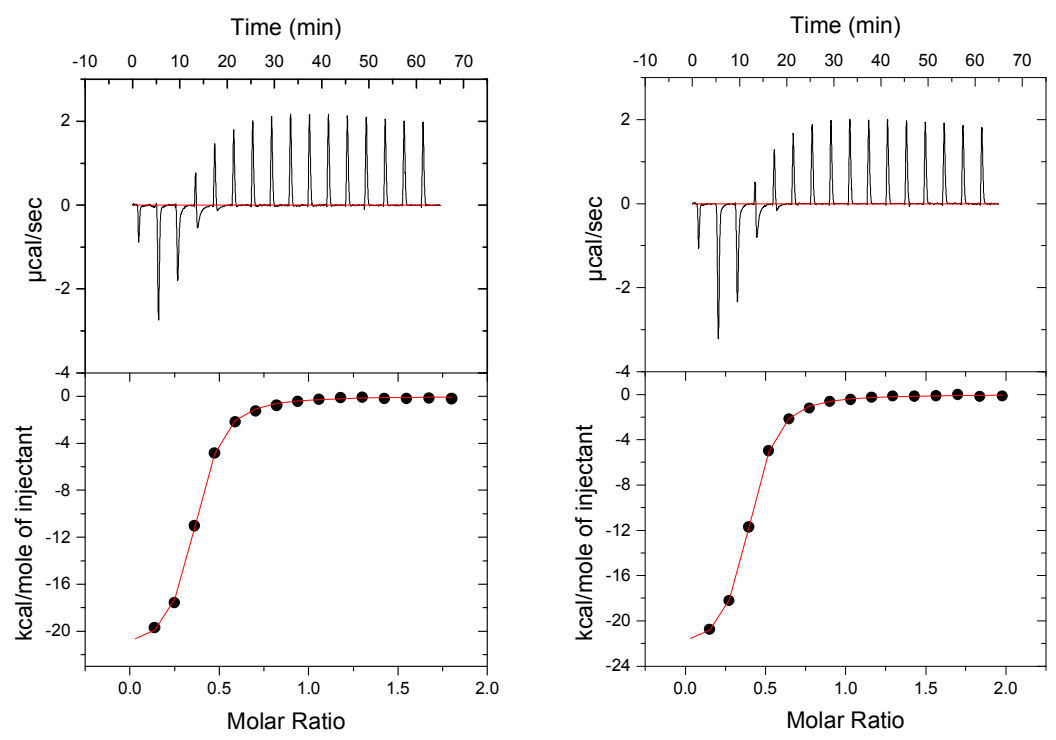


\section{Supporting material for entries depicted in table 3}

a) ITC traces of the titration of open-chain 3 with $(\boldsymbol{R}, \boldsymbol{R})-\operatorname{tartrate}^{2-} \mathbf{3 2}$ (left) and $(\boldsymbol{S}, \boldsymbol{S})-\operatorname{tartrate}^{2-} \mathbf{3 1}^{-}$ (right). Titration mode: host into guest (tetraethylammonium salt) solution; two-site-model.
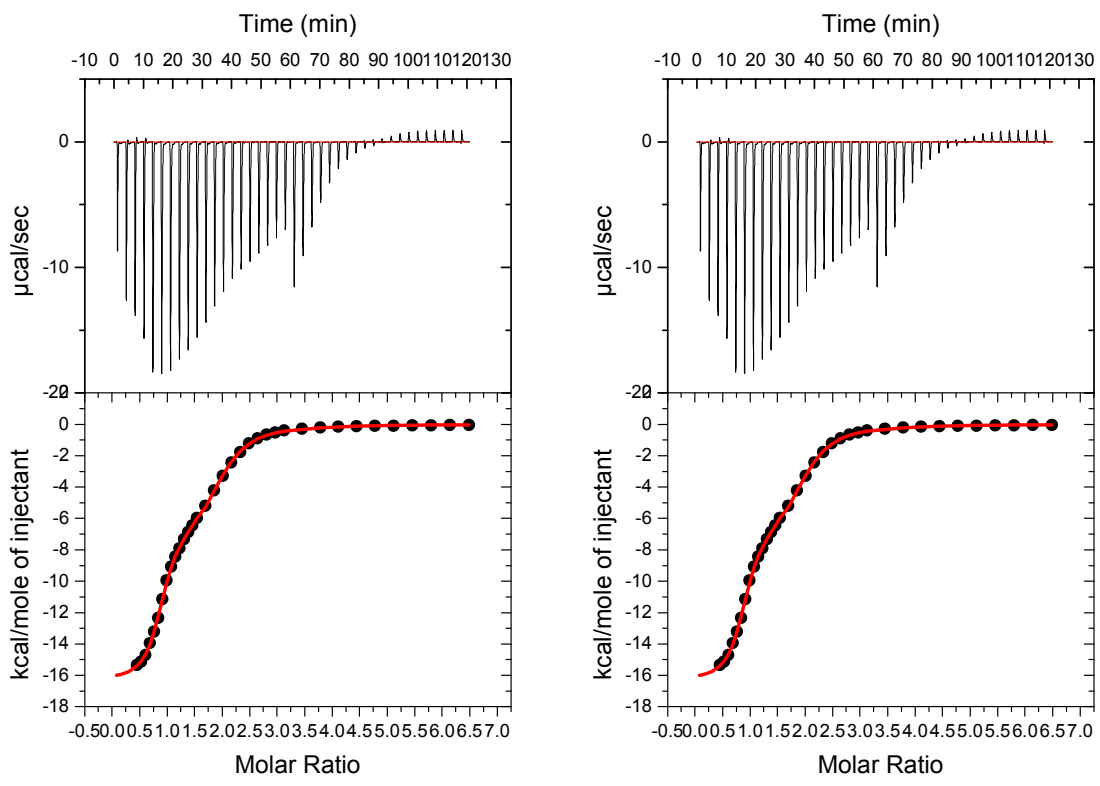

b) ITC traces of the titration of open-chain 3 with $\boldsymbol{R}$-aspartate ${ }^{\mathbf{1}^{-}} \mathbf{3 3}^{-}$(left) and $\boldsymbol{S}$-aspartate $\mathbf{1}^{\mathbf{1}^{-}} \mathbf{3 4}$ (right). Titration mode: host into guest (tetraethylammonium salt) solution; one-site-model; ligand-incell
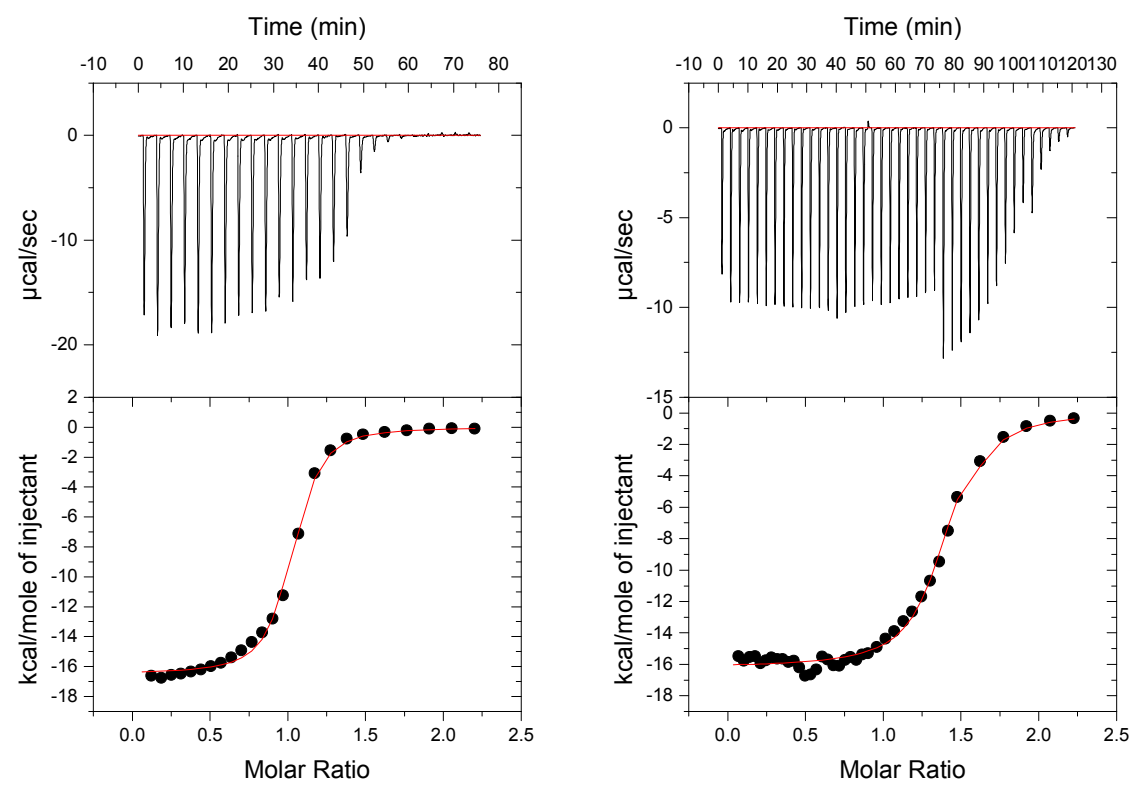
c) ITC traces of the titration of open-chain 3 with $\boldsymbol{R}$-phenylglycine ${ }^{1-} \mathbf{3 7}$ (left) and $\boldsymbol{S}$ phenylglycine $^{1-} 38$ (right). Titration mode: host into guest (tetraethylammonium salt) solution; one-site-model; ligand-in-cell
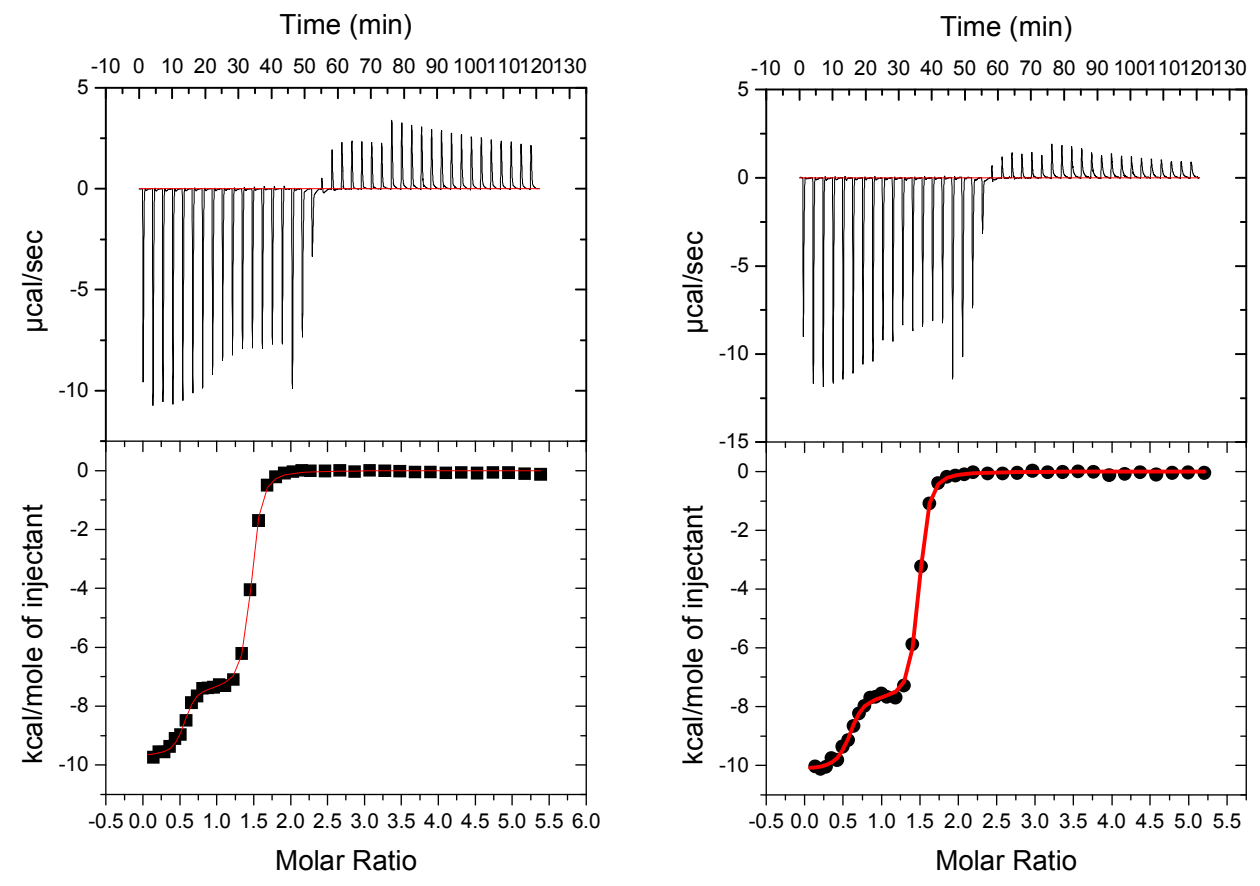